This item was submitted to Loughborough's Research Repository by the author.

Items in Figshare are protected by copyright, with all rights reserved, unless otherwise indicated.

\title{
Dynamical phenomena connected with stability loss of equilibria and periodic trajectories
}

PLEASE CITE THE PUBLISHED VERSION

https://doi.org/10.1070/RM10023

PUBLISHER

Turpion

VERSION

SMUR (Submitted Manuscript Under Review)

\section{PUBLISHER STATEMENT}

This paper was submitted for publication in the journal Russian Mathematical Surveys and the definitive published version is available at https://doi.org/10.1070/RM10023.

LICENCE

CC BY-NC-ND 4.0

\section{REPOSITORY RECORD}

Neishtadt, Anatoly, and Dmitry Treschev. 2021. "Dynamical Phenomena Connected with Stability Loss of Equilibria and Periodic Trajectories". Loughborough University. https://hdl.handle.net/2134/16661419.v1. 


\title{
Dynamical phenomena connected with stability loss of equilibriums and periodic trajectories
}

\author{
A.I.Neishtadt* $\quad$ D.V.Treschev ${ }^{\dagger \ddagger}$
}

\begin{abstract}
We study a dynamical system depending on a parameter $\kappa$. Assuming that the system has a family of equilibrium positions or periodic trajectories smoothly depending on $\kappa$, we are interested in details of stability loss through various bifurcations (Poincaré-Andronov-Hopf, period doubling, etc.). We deal with two basic set ups. In the first one $\kappa$ is constant and the subject of the analysis is the phenomenon of a soft and hard loss of stability. In the second set up $\kappa$ slowly depends on time (the case of a dynamical bifurcation). In the simplest situation $\kappa=\varepsilon t$, where $\varepsilon$ is a small parameter. More generally, $\kappa(t)$ may be a solution of a slow differential equation. In the case of a dynamical bifurcation the analysis is concentrated basically around the phenomenon of the stability loss delay.
\end{abstract}

\section{Introduction}

Dynamical stability of equilibrium positions and periodic solutions presents one of the first problems in qualitative theory of differential equations. Exact definition of dynamical stability for a solution of an ordinary differential equation (ODE) is given in [46] although informally this concept was known long before Lyapunov. Below the terms "dynamical stability" and "Lyapunov stability" mean the same. Generically Lyapunov stability follows from linear stability i.e., stability of the corresponding linearized system. From this viewpoint Hamiltonian systems are not generic.

In Hamiltonian case the linear stability does not imply directly the Lyapunov stability. One of the origins of the dynamical stability of an equilibrium position $x_{0}$ is the positive definiteness of the Hamiltonian function at $x_{0}$. More precisely, Lagrange-Dirichlet theorem deals with the so-called natural Hamiltonians which have the form of the sum of a kinetic energy (a positive definite quadratic form in the momenta $p$ ) and a potential $V$ (a function of the coordinates $q$ only). The theorem says that if $V$ has an isolated minimum at $q_{0}$ then the equilibrium position $(p, q)=\left(0, q_{0}\right)$ is dynamically stable.

A great activity was performed to prove the inverse statement: if $q_{0}$ is an isolated critical point which is not a local minimum then the equilibrium is unstable. At the

\footnotetext{
*Loughborough University, UK and Space Research Institute of Russian Academy of Sciences.

${ }^{\dagger}$ Steklov Mathematical Institute of Russian Academy of Sciences and Lomonosov Moscow State University.

${ }^{\ddagger}$ The second author was supported by the Russian Science Foundation under grant 20-11-20141.
} 
moment this statement is proven only in the case of 2 degrees of freedom for real-analytic potentials [60]. Many references and results for an arbitrary number of degrees of freedom can be found in [40].

If an equilibrium position $x_{0}$ is not a minimum or maximum of the Hamiltonian function, proof of Lyapunov stability for linearly stable equilibrium position (based on arguments from KAM-theory) can be obtained only in the case of not more than two degrees of freedom.

Recall that KAM-theory present theorems on preservation of invariant tori of integrable Hamiltonian systems after a small perturbation. In a small neighborhood $U$ of an equilibrium position or a periodic orbit any Hamiltonian system is close to linear one and therefore, under some non-degeneracy assumptions, $U$ contains a subset of a large relative measure of invariant tori. In the case of 2 degrees of freedom these tori have codimension 1 on the 3-dimensional energy levels. Hence, they divide these levels into invariant domains. Combination of this observation with simple geometric arguments concerning relative position of these domains and the equilibrium (periodic orbit) imply Lyapunov stability.

If the dimension of the system is higher, Lyapunov stability can be broken by Arnold diffusion. The corresponding discussion is presented in [6]. It is however, not proven at the moment that the break down of the Lyapunov stability due to Arnold diffusion is typical. ${ }^{1}$ In this context we mention interesting recent results and historical comments presented in [13, 26], and [25].

We consider systems of ordinary differential equations depending on a parameter

$$
\dot{x}=f(x, \kappa), \quad x \in \mathcal{X}, \quad \kappa \in \mathcal{K} \subset \mathbb{R}^{k} .
$$

Here $\mathcal{X}$ is a smooth manifold, usually a domain in $\mathbb{R}^{n}$. First, we assume that $\kappa$ is constant while in the second part of the paper we consider $\dot{\kappa} \sim \varepsilon$ (in particular, $\kappa=\varepsilon t$ ), where $\varepsilon>0$ is small. The function $f: \mathcal{X} \times \mathcal{K} \rightarrow \mathbb{R}^{n}$ is smooth. In some situations we will need real-analyticity in $x, \kappa$.

Suppose that for any value of $\kappa \in \mathcal{K}$ the system has an equilibrium position $x_{\kappa} \in \mathcal{X}$ smoothly depending on $\kappa$. It is natural to consider linearization of system (1.1) at $x_{\kappa}$ :

$$
\dot{\xi}=A_{\kappa} \xi, \quad \xi=x-x_{\kappa}, \quad A_{\kappa}=\frac{\partial f}{\partial x}\left(x_{\kappa}, \kappa\right) .
$$

Lyapunov stability of the equilibrium $x_{\kappa}$ is essentially determined by the spectrum of the operator $A_{\kappa}$. If all eigenvalues of $A_{\kappa}$ have negative real parts then $x_{\kappa}$ is asymptotically stable. If there exists an eigenvalue with positive real part, $x_{\kappa}$ is exponentially unstable. If all real parts of the eigenvalues are nonpositive but there exist purely imaginary eigenvalues (a situation typical for Hamiltonian systems), the problem of stability becomes more complicated.

Analogous situation appears if instead of equilibrium positions $x_{\kappa}$ we have a smooth family of periodic trajectories $\gamma_{\kappa} \subset \mathcal{K}$. In this case Lyapunov stability of any $\gamma_{\kappa}$ depends on its multipliers, the eigenvalues of the monodromy operator. If all the multipliers are inside the unit circle then $\gamma_{\kappa}$ is asymptotically stable. If there exists a multiplier with

\footnotetext{
${ }^{1}$ In real-analytic category this is an especially complicated problem.
} 


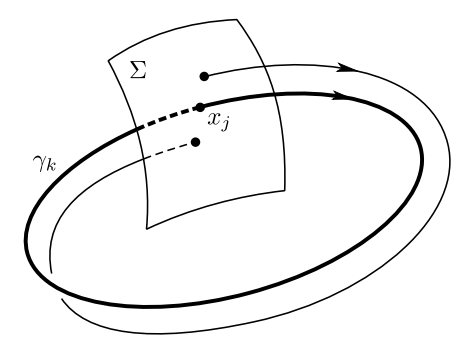

Figure 1: Periodic trajectory and the Poincaré map

absolute value greater than one, we have exponential instability. Stability in Hamiltonian case is again a more delicate problem.

Poincaré noticed that in many cases it is more convenient to study the dynamics in a neighborhood of a periodic solution $\gamma$ by using reduction to a system with discrete time. This can be done locally taking a (small) hypersurface $\Sigma \subset \mathcal{X}$ transversal to the vector field $f$ so that $\gamma_{\kappa}$ intersects $\Sigma$ at one point $x_{\gamma}$. Then on some (may be, smaller) hypersurface $\Sigma_{1} \subset \Sigma$ the first return map is well defined:

$$
F: \Sigma_{1} \rightarrow \Sigma, \quad F\left(x_{\gamma}\right)=x_{\gamma}
$$

see Fig. 1. Hence, the periodic trajectory $\gamma$ for system (1.1) is replaced by the fixed point $x_{\gamma}$ for the map $F$.

There is an inverse standard construction, called a suspension, which associates with a diffeomorphism $F$ (a dynamical system with discrete time) an ODE system (a system with continuous time). If $F$ is defined on a smooth manifold $M$, we consider the vector field $f=\partial / \partial t$ on the direct product $M \times[0,1]$, where $t$ is the coordinate on the interval $[0,1]$. The phase space of the ODE is $\widetilde{M}=M \times[0,1] / \sim$, where the identification $\sim$ is as follows:

$$
x \times\{0\} \sim F(x) \times\{1\} \quad \text { for any } x \in M .
$$

Problems and dynamical effects are easily transformed from systems with continuous time to the case of a discrete time. In general it is little known about topology of $\widetilde{M}$. Sometimes it is important to deal with the phase space diffeomorphic to $M \times \mathbb{T}$, where $\mathbb{T}$ is a circle. In this case the suspension construction should be replaced by something more complicated. The corresponding discussion can be found in [80], see a more resent result in [81].

We assume that $\mathcal{K}$ contains both: stable and unstable equilibriums (periodic solutions). In particular, if $\mathcal{K} \subset \mathbb{R}$ is an interval containing zero, we assume that $x_{\kappa}$ (respectively, $\gamma_{\kappa}$ ) is stable for $\kappa<0$ and unstable for $\kappa>0$. Hence, at the point $\kappa=0$ loss of stability takes place. We start from two simplest examples. In both cases $\kappa$ is one-dimensional.

Example 1. Let $\mathcal{X} \subset \mathbb{R}^{2}$ be a domain and let $x_{\kappa}$ be a family of fixed points. Suppose that the loss of stability appears when two complex conjugate eigenvalues $\lambda(\kappa)$ and $\bar{\lambda}(\kappa)$ of the matrix $A_{\kappa}$ pass through the imaginary axis:

$$
\lambda(0) \in i \mathbb{R}, \quad 0<\operatorname{Im} \lambda(0),\left.\quad \frac{d}{d \kappa}\right|_{\kappa=0} \operatorname{Re} \lambda(\kappa)>0 .
$$



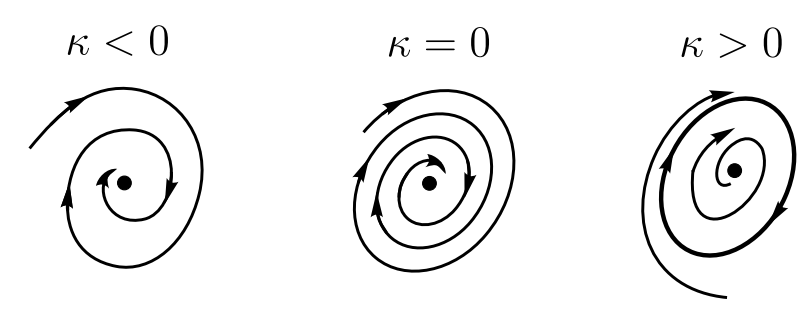

Figure 2: Poincaré-Andronov-Hopf bifurcation, soft loss of stability.

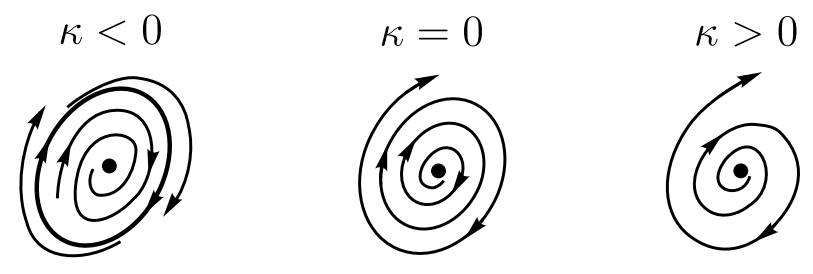

Figure 3: Poincaré-Andronov-Hopf bifurcation, hard loss of stability.

This situation is known as the Poincaré-Andronov-Hopf bifurcation $[62,1,2] .{ }^{2}$ Generically, depending on the sign of a certain coefficient in the normal form, there are two possibilities. When $\kappa$ increases and passes through zero

(a) at the point $x(0)$ an asymptotically stable limit cycle $\mathcal{C}_{\kappa}$ is born, its size is of order $\sqrt{\kappa}$ (the soft loss of stability), see Fig. 2.

(b) at $x(0)$ an exponentially unstable limit cycle $\mathcal{C}_{\kappa}$ dyes, its size is of order $\sqrt{-\kappa}$ (the hard loss of stability), see Fig. 3.

In the case of the soft stability loss the equilibrium position $x(0)$ is Lyapunov stable and for $\kappa>0$ its stability is transmitted to a small limit cycle: solutions with initial conditions close to $x_{\kappa}$ stay in a small neighborhood of the (unstable) equilibrium and approach the cycle $\mathcal{C}_{\kappa}$.

In the case of the hard stability loss the stability domain for negative $\kappa$ has the diameter of order $\sqrt{-\kappa}$ and for any small $\kappa \geq 0$ solutions with almost all initial data near $x_{\kappa}$ leave a fixed neighborhood of the point $x(0)$.

Multidimensional generalizations of this dynamical picture are straightforward if we assume that for $\kappa$ close to 0 real parts of all other eigenvalues stay negative.

Example 2. Consider the local diffeomorphism $F_{0}$ of the interval $I \subset \mathbb{R}$ with the fixed point 0 and the multiplier -1 :

$$
F_{0}: I_{1} \subset I \rightarrow I, \quad F_{0}(x)=-x+O\left(x^{2}\right) .
$$

Note that in this case the suspension construction gives a non-orientable phase space, the Möbius strip ${ }^{3}$. In higher dimensional analogs of Example 2 orientable phase spaces

\footnotetext{
${ }^{2}$ Here we deem appropriate to quote Arnold, [5]: "In works on the catastrophe theory the soft stability loss of the equilibrium position is usually referred to as the Hopf bifurcation (partially by my "fault" because, exposing the Poincaré-Andronov theory to R.Thom in 1965, I particularly emphasized on the work of E.Hopf who had extended a part of this theory to the multidimensional case)".

${ }^{3}$ because $F$ changes orientation on $I$
} 
appear as well as non-orientable ones.

Now consider a perturbation $F_{\kappa}(x)=F_{0}(x)+O(\kappa)$. By using a change of the variable

$$
x=y+O\left(|\kappa|+y^{2}\right),
$$

it is easy to present the map in the form

$$
y \mapsto \mathcal{F}_{\kappa}(y)=-(1+a(\kappa)) y+A(\kappa) y^{3}+O\left(y^{4}\right), \quad a(0)=0 .
$$

In this way we transform the family of fixed points $x_{\kappa}$ to $\{y=0\}$ and make $\mathcal{F}_{\kappa}$ free of quadratic in $y$ terms.

We assume the nondegeneracy condition $a^{\prime}(0) A(0) \neq 0$. Suppose for definiteness that $a^{\prime}(0)>0$. Then for small $\kappa<0$ the fixed point $y=0$ is asymptotically stable while for $\kappa>0$ it is unstable. We have to distinguish two cases: positive and negative $A(0)$.

(a) Suppose $A(0)>0$. Then for small $\kappa \leq 0$ the stable fixed point $y=0$ attracts all trajectories from $U_{c}$. For $\kappa>0$ a stable orbit of period 2 of form (1.3) appears which attracts all trajectories starting in $U_{c}$ except the (unstable) fixed point $y=0$. This situation corresponds to the soft stability loss and is usually called the period doubling bifurcation.

(b) Suppose $A(0)<0$. Then for $\kappa<0$ the system has an unstable periodic orbit of period 2

$$
y= \pm \sqrt{\kappa a^{\prime}(0) / A(0)}+O(\kappa)
$$

which bounds the stability zone around the origin. The size of this zone is of order $\sqrt{-\kappa}$.

For $\kappa>0$ trajectories started near the point $y=0$ leave a neighborhood $U_{c}=\{|y|<c\}$ for some $c>0$, independent of $\kappa$. This situation corresponds to the hard stability loss.

Examples 1 and 2 are typical for the static bifurcation theory. In this theory a dynamical system, which depends on a parameter $\kappa=$ const, is considered in a neighbourhood of a critical (bifurcational) value of the parameter. In the theory of dynamical bifurcations parameter changes slowly in time and passes through a value which is critical in the static theory.

Initial view on this subject was that in the principal approximation it is enough to consider the problem quasi-statically, assuming that with the slow change of the parameter the system switches from an unstable to a stable state almost instantly when passing through a bifurcation. The role of the theory of dynamical bifurcations would be then to justify this point of view by supplying estimates related to passages suggested by the quasi-static theory. This point of view is correct in many cases. It was a big surprise to discover that there are rather general situations where dynamics is completely different from the predictions of the quasi-static approach.

The theory of dynamical bifurcations deals with slow-fast dynamical systems: the slow-fast ODE systems

$$
\dot{x}=f(x, \kappa, \varepsilon), \quad \dot{\kappa}=\varepsilon g(x, \kappa, \varepsilon), \quad x \in \mathcal{X}, \quad \kappa \in \mathcal{K} \subset \mathbb{R}^{k} .
$$

and slow-fast maps

$$
x \mapsto f(x, \kappa, \varepsilon), \quad \kappa \mapsto \kappa+\varepsilon g(x, \kappa, \varepsilon), \quad x \in \mathcal{X}, \quad \kappa \in \mathcal{K} \subset \mathbb{R}^{k} .
$$


Here $\varepsilon, 0<\varepsilon \ll 1$ is a small parameter which characterises the rate of slow change of $\kappa, x$ is called a fast variable, $\kappa$ is called a slow variable. For $\varepsilon=0$ we obtain a family of differential equations (or maps) for $x$ which is called a fast equation or a fast system (respectively, a fast map).

Study of dynamical bifurcations was started in relation to works on relaxation oscillations (see [49] and references therein). Consider a slow passage through the so-called tangent bifurcation of equilibrium. At critical values of slow variables the fast equation has an equilibrium with one zero eigenvalue while all other eigenvalues have negative real parts. These critical values form a surface in the space of slow variables. Two equilibria of the fast equation, stable and unstable one, merge and disappear when values of slow variables pass transversally through this surface. The estimates describing dynamics in the whole slow-fast system in this case are obtained under the condition that this critical surface is indeed crossed transversally. The phase points leave a neighbourhood of the equilibria.

It was a rather unexpected discovery that, when the transversality condition is dropped, the phase points can switch from the motion near stable equilibrium position to the motion near the unstable one and stay in this mode for a long time $\sim 1 / \varepsilon$ [14]. This happens generically if the system depends on an additional parameter. The phenomenon takes place in an exponentially small $(O \exp (-$ const $/ \varepsilon))$ interval of this parameter. The corresponding phase trajectories are known as "canard" trajectories. This work was continued in different directions (see, e.g., [11, 18] and references therein). The subject is known under the colourful name "chasse au canard".

We consider another dynamical bifurcation phenomenon, which looks similar to a "canard" (a long motion near an unstable equilibrium or a periodic trajectory of the fast system, near an unstable fixed point of the fast map), but has a completely different origin. This phenomenon, usually refereed to as a stability loss delay, is related to slow passage through a bifurcation, corresponding to stability loss of a non-degenerate equilibrium or a non-degenerate periodic trajectory of a fast system, or a non-degenerate fixed point of a fast map. It takes place in any analytic system with passage through such a bifurcation [53].

There is so called "trivial stability loss delay" which takes place when the equilibrium (or a fixed point, or a periodic trajectory) of the fast system is the same for all values of slow variables. This is a case of an infinite degeneration (some function should vanish identically).

Theory of stability loss delay was started by the paper [69], where the first example of this phenomenon in the non-trivial (non-degenerate) situation was given. This example was analysed by considering the dynamics in the complex time. Such an approach and its modifications later turned out to be a very general and powerful tool in analysis of this phenomenon. Some generalisations of the example from [69] are contained in [37]. Existence of a (non-trivial) stability loss delay can be derived also from results of [71]. Development of a general theory of the stability loss delay phenomenon was started in $[52,53,54]$.

The structure of the paper is as follows. In the first part (Sections 2-3) we study loss of stability in Hamiltonian systems in the framework of static bifurcation theory. Here two possibilities are typical: hard and soft stability loss. 
In Section 2 we present results on Hamiltonian period doubling bifurcation. In Section 3 we deal with Hamiltonian Poincaré-Andronov-Hopf bifurcation. Qualitatively these results are similar. In the case of a soft stability loss solutions, starting in an $\varepsilon$-neighborhood $U_{\varepsilon}$ of the trajectory $\gamma_{\kappa}, \kappa \geq 0$ (respectively, the equilibrium position $x_{\kappa}$ ), stay in the domain $U_{c \sqrt{\varepsilon+\kappa}}$. This is true for all such solutions if the number of degrees of freedom is at most 2 while for systems with more than two degrees of freedom this is true for a big relative measure of initial conditions in $U_{\varepsilon}$. In the case of a hard stability loss for any positive $\kappa$ and $\varepsilon$ there exists a solution with initial conditions in $U_{\varepsilon}$ which leaves the domain $U_{c}$, where $c>0$ is independent of $\kappa$ and $\varepsilon$.

The second part of the paper (Sections 4-7) concerns dynamical bifurcations. We assume that $f$ and $g$ in (1.4), (1.5) are at least twice continuously differentiable in $x, \kappa$ and once continuously differentiable in $\varepsilon$ for $(x, \kappa) \in \mathcal{X} \times \mathcal{K}$ and $|\varepsilon|<\varepsilon_{1}$ where $\varepsilon_{1}=$ const $>0$. For the slow-fast system (1.4) we consider two cases: a) the fast equation has an equilibrium which is non-degenerate and depends continuously of $\kappa$, and b) the fast equation has a periodic trajectory which is non-degenerate and depends continuously of $\kappa$. For the slow-fast map (1.5) we assume that the fast map has a fixed point which is non-degenerate and depends continuously of $\kappa$. To have short formulations, we call $a$ state $L_{\kappa}$ each of these objects embedded into $\mathcal{X} \times \mathbb{R}^{k}$. We introduce the slow equation

$$
\dot{\kappa}=\varepsilon G(\kappa) .
$$

If the state $L_{\kappa}$ is an equilibrium of the fast equation or a fixed point of the fast map, then $G(\kappa)$ is the value of $g(x, \kappa, 0)$ at this state. If $L_{\kappa}$ is a periodic trajectory of the fast equation, then $G(\kappa)$ is the time average of $g(x, \kappa, 0)$ along $L_{\kappa}$.

For $\varepsilon>0$ we introduce the slow time $\tau=\varepsilon t$. Let

$$
\kappa=K(\tau), \quad \tau_{0} \leq \tau \leq \tau_{1}, \quad K\left(\tau_{0}\right)=\kappa_{0} .
$$

be a solution of the slow equation. Suppose that there is $\tau_{*} \in\left(\tau_{0}, \tau_{1}\right)$ such that the state $L_{K(\tau)}$ of the fast equation (or of the fast map) is asymptotically stable in the linear approximation for $\tau_{0} \leq \tau<\tau_{*}$ and loses stability at $\tau=\tau_{*}$, but remains non-degenerate. In the case of an equilibrium, at $\tau=\tau_{*}$ this equilibrium has a pair of complex conjugate purely imaginary eigenvalues, and 0 is not an eigenvalue. In the case of a periodic trajectory (or a fixed point) this state has either a pair of complex conjugate multipliers on the unit circle, or the multiplier $(-1)$, and 1 is not a multiplier.

In Section 4 we consider stability loss in the above context from the viewpoint of real time analysis. We show that stability loss delay is an unavoidable phenomenon for analytic systems.

In Sections 5-6 we consider stability loss delay of equilibria from the viewpoint of complex time analysis. Section 5 is devoted to the case when paths in complex time are bounded away from singularities. An asymptotic formula for delay time is given. In Section 6 it is shown how passage through a singularity in complex time leads to a remarkable buffer point phenomenon. In Section 7 we discuss stability loss delay of periodic trajectories from the viewpoint of complex time analysis. 


\section{Hamiltonian period doubling}

\subsection{Theorem on the stability loss}

Consider a Hamiltonian system with one and a half degrees of freedom.The Hamiltonian is a function of one coordinate, one momentum and time $t \bmod 2 \pi$. Systems of such type appear, for example, after the isoenergetic reduction from Hamiltonian systems with two degrees of freedom in a neighborhood of a periodic trajectory. We also assume that the Hamiltonian function depends on the parameter $\kappa$ which takes values in an interval $\left(-\kappa_{0}, \kappa_{0}\right) \subset \mathbb{R}$.

Let $\gamma_{\kappa}$ be a smooth in $\kappa$ family of non-degenerate $2 \pi$-periodic trajectories. Linear stability of any orbit $\gamma_{\kappa}$ is determined by its multipliers $\lambda^{ \pm 1}(\kappa)$, where $\lambda \neq 1$ is a smooth function of $\kappa$. If $\lambda \in \mathbb{R} \backslash\{ \pm 1\}$, the periodic solution is called hyperbolic. If $\lambda(\kappa)$ is not real, we have $|\lambda|=1$. In this case $\gamma_{\kappa}$ is said to be elliptic.

We assume that $\lambda(\kappa)$ is elliptic for $\kappa<0$, hyperbolic for $\kappa>0$, and for $\kappa=0$ we have $\lambda=-1$ (parabolic case). Hence, when $\kappa$ passes through 0 , the multipliers $\lambda^{ \pm 1}(\kappa)$ collide at -1 and drift away along the real axis, initiating loss of stability for $\gamma_{\kappa}$.

To study the stability loss of $\gamma_{\kappa}$ when $\kappa$ increases and passes through zero, we use convenient coordinates in a neighborhood of the trajectory $\gamma_{0}$. By using a $4 \pi$-periodic change of the phase variables, we present the system in the form [6]

$$
\begin{gathered}
\dot{p}=-\partial H_{\kappa} / \partial q, \quad \dot{q}=\partial H_{\kappa} / \partial p, \\
H_{\kappa}(p, q, t)=H_{*}+O\left(p^{3}, q^{5}, p q^{3}, p^{2} q\right), \quad H_{*}=-\delta q^{2}+p^{2} / 2+D q^{4} .
\end{gathered}
$$

Here $\delta$ and $D$ are smooth functions of $\kappa$,

$$
\delta(0)=0, \quad \delta(\kappa) / \kappa \geq 0, \quad \gamma_{\kappa}=\{(p, q, t \bmod 4 \pi): p=q=0\}
$$

The function $H_{*}$ is said to be the normal form. Phase portraits of the normal form are presented in Fig. 4. We assume the non-degeneracy conditions

$$
d \delta /\left.d \kappa\right|_{\kappa=0}>0, \quad D(0) \neq 0 .
$$

For any $\kappa \in\left(-\kappa_{0}, \kappa_{0}\right)$ we define the family of neighborhoods $U_{r}=U_{r}(\kappa)$ of $\gamma_{\kappa}$

$$
U_{r}=\{(p, q, t \bmod 4 \pi): \rho \leq r\}, \quad \rho^{2}=p^{2}+q^{2} .
$$

Theorem 1 (a). Suppose $D(0)>0$. Then the stability loss is soft: there exists a constant $c>0$ such that for small nonnegative $r$ and $\kappa$ any solution with initial data in $U_{r}(\kappa)$ does not leave the domain $U_{c \sqrt{\kappa+r}}(\kappa)$.

(b). Suppose $D(0)<0$. Then the stability loss is hard: for any small $r>0$ and $\kappa \geq 0$ the domain $U_{r}(\kappa)$ contains initial data such that the corresponding solution leaves $U_{c}(\kappa)$ for some $c>0$ independent of $r$ and $\kappa$.

Remark 2.1 1. If $D(0)<0$ and $\kappa>0$ then almost all solutions starting in $U_{r}(\kappa)$, leave $U_{c}(\kappa)$. Proof of this stronger version of assertion (b) needs a construction of asymptotic manifolds for $\gamma_{\kappa}, \kappa \geq 0$. We expect that the corresponding argument is essentially close to standard. 
$D>0$
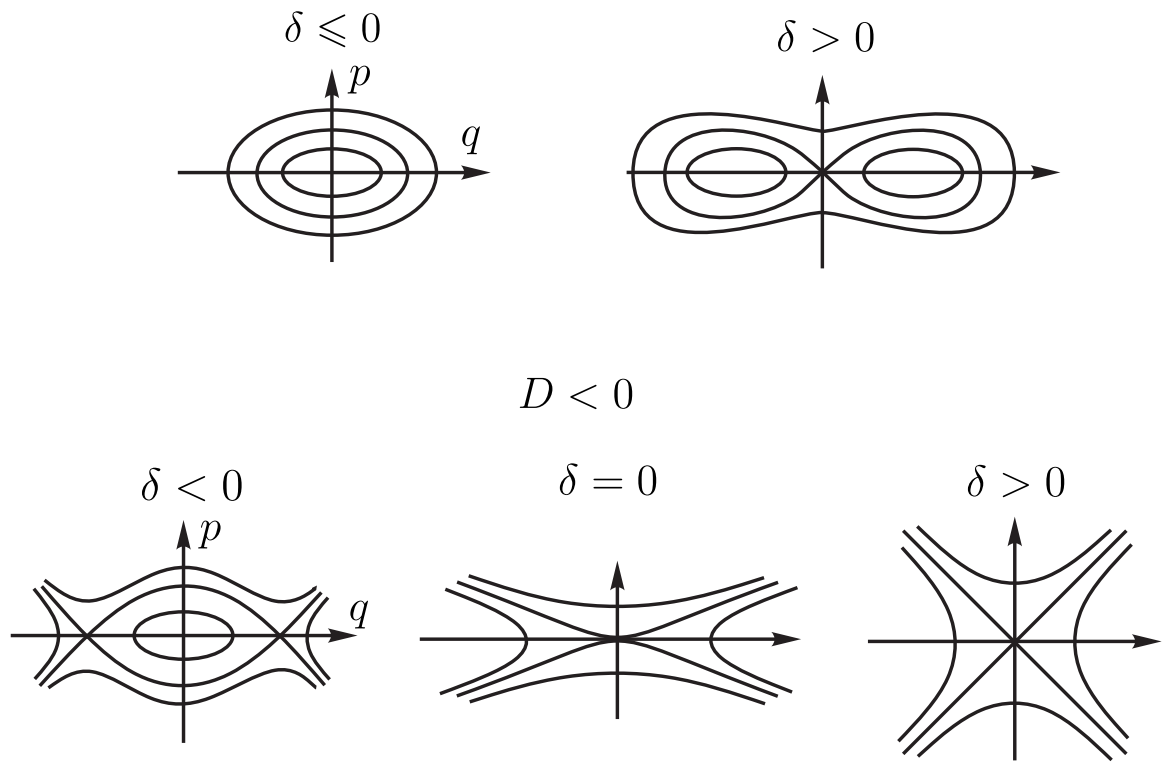

Figure 4: Level lines of the Hamiltonian $H_{*}$

2. Theorem 1 admits an obvious multi-dimensional extention in the case of the presence of additional variables which generate multipliers lying on the unit circle. Here it is necessary to keep in mind that a convenient normal form requires absence of low order resonances and replace the Lyapunov stability by the stability for majority of initial conditions.

\subsection{Proof of Theorem 1}

(a) First, we estimate the diameter $d=d(r)$ of the domain $U_{d}$ which contains all trajectories of the system with Hamiltonian $H_{*}$ starting in $U_{r}$.

Lemma 2.1 Suppose $D(0)>0$. If $r$ and $\delta$ are sufficiently small then for some $c_{0}>0$, $c_{0}=c_{0}(\delta(0), D(0))$

$$
d \leq c_{0} \sqrt{\kappa+r}
$$

Proof of Lemma 2.1. Any trajectory of the system with Hamiltonian $H_{0}$ lies in

$$
Q_{h}=\left\{(p, q, t \bmod 2 \pi): H_{0}(p, q, t)=h\right\} .
$$

Hence, we can take $d^{2}(r)=\max _{Q_{h}} \rho^{2}$, where $h$ satisfies the condition $\min _{Q_{h}} \rho^{2} \leq r^{2}$.

By the Lagrange principle at critical points $(p, q)$ of $\rho^{2}$ on $Q_{h}$ the covectors $\operatorname{grad} \rho^{2}$ and $\operatorname{grad} H_{0}$ are linearly dependent i.e., ${ }^{4}$

$$
\operatorname{rank}\left(\begin{array}{cc}
-2 \delta q+4 D q^{3} & p \\
q & p
\end{array}\right)<2
$$

\footnotetext{
${ }^{4}$ For brevity we write $D$ instead of $D(0)$.
} 
If $p$ and $q$ are small, this condition holds in two cases: $q=0$ and $p=0$.

If $q=0$, we have $p^{2} / 2=h$ while for $p=0$ we have: $-\delta q^{2}+D q^{4}=h$. The corresponding critical values of $\rho^{2}$ are

$$
\rho^{2}=\rho_{0}^{2}:=2 h \quad \text { and } \quad \rho^{2}=\rho_{ \pm}^{2}:=\frac{\delta \pm \sqrt{\delta^{2}+4 h D}}{2 D} .
$$

If $h$ is a (small) positive number then $\rho_{0}$ is the minimum, $\rho_{+}$is the maximum while $\rho_{-}^{2}$ is negative and should be ignored. The inequality $2 h \leq r^{2}$ implies

$$
d^{2}=\frac{\delta+\sqrt{\delta^{2}+4 h D}}{2 D} \leq \frac{\sqrt{\delta^{2}+4 h D}}{D} \leq \frac{\delta+\sqrt{4 h D}}{D} \leq \frac{\delta+\sqrt{2 D} r}{D} .
$$

We obtain (2.3) because by $(2.2) \delta \leq 2 D \kappa$.

If $h$ is negative, $\rho_{0}$ should be ignored while $\rho_{ \pm}^{2} \leq \delta / D$. Therefore $d \leq \sqrt{\delta / D}$. This also implies (2.3).

In a neighborhood of $\gamma_{\kappa}$ the system (2.1) is a small perturbation of the integrable system with Hamiltonian $H_{*}$. Consider the invariant torus

$$
\mathbb{T}_{h}^{2}=\left\{(p, q, t \bmod 4 \pi): H_{*}=h\right\}
$$

of the unperturbed system. It is sufficient to consider $h \geq \delta^{2}$. We put $h=(\delta+\beta)^{2}, \beta \geq 0$. In the new coordinates

$$
q=\sqrt{\delta+\beta} \hat{q}, \quad p=(\delta+\beta) \hat{p}, \quad H_{\kappa}=(\delta+\beta)^{3 / 2} \hat{H}
$$

the system (2.1) has the form

$$
\begin{gathered}
\dot{\hat{p}}=-\partial \hat{H} / \partial \hat{q}, \quad \dot{\hat{q}}=\partial \hat{H} / \partial \hat{p} \\
\hat{H}(\hat{p}, \hat{q}, t)=\sqrt{\delta+\beta} \hat{H}_{*}+O(\delta+\beta), \quad \hat{H}_{*}=\left(-\frac{\delta}{\delta+\beta} \hat{q}^{2}+\hat{p}^{2} / 2+D(0) \hat{q}^{4}\right) .
\end{gathered}
$$

and the torus $\mathbb{T}_{h}^{2}$ is

$$
\mathbb{T}_{h}^{2}=\left\{(\hat{p}, \hat{q}, t \bmod 4 \pi): \hat{H}_{*}=1\right\} .
$$

For majority of small $h \geq \delta^{2}$ this torus is Diophantine and non-degenerate.

Note that in the system (2.4) only one variable is fast, the angle $t$, while the others, $\hat{p}$ and $\hat{q}$ have time derivatives $\sim \sqrt{\delta+\beta}$. In such a situation by using a change of variables, the time dependence in the Hamiltonian $\hat{H}$ can be considerably reduced. More precisely, there exists a near-identity canonical $4 \pi$-periodic in $t$ change of coordinates

$$
\hat{p}=\tilde{p}+O(\sqrt{\delta+\beta}), \quad \hat{q}=\tilde{q}+O(\sqrt{\delta+\beta})
$$

such that the Hamiltonian takes the form

$$
\tilde{H}(\tilde{p}, \tilde{q}, t)=\sqrt{\delta+\beta} \hat{H}_{*}(\tilde{p}, \tilde{q})+(\delta+\beta) \tilde{H}_{1}(\tilde{p}, \tilde{q}, \delta, \beta)+\mathcal{H}(\tilde{p}, \tilde{q}, t),
$$

where $\mathcal{H}=O\left((\delta+\beta)^{N}\right)$ for arbitrary $N$ if the function $H$ is smooth and $O\left(e^{-\tilde{c} / \sqrt{\delta+\beta}}\right)$ for some $\tilde{c}>0$ if $H$ is real-analytic, [51], see also [80]. This remark is not necessary for the 


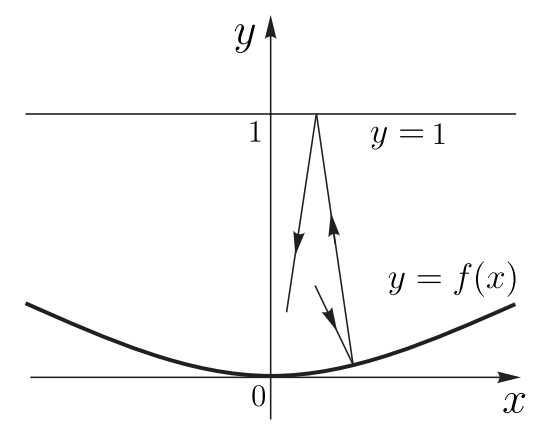

Figure 5: Billiard system in a domain, bounded by the curves 2.5 .

further argument and just means that the chaotic zones in system (2.4) are much smaller than one could expect having in $\hat{H}$ the perturbation of order $O(\delta+\beta)$.

According to KAM-theory majority of tori $\mathbb{T}_{h}^{2}$ survive the perturbation. They lock trajectories in narrow slots called the Birkhoff instability zones. Due to this the estimate (2.3) remains valid with a little bit bigger constant instead of $c_{0}$.

(b) To prove instability of $\gamma_{\kappa}$ in the case $D(0)<0$, we consider the function $V=p q$. Its derivative along solutions of system (2.1) equals

$$
d V / d t=2 \delta q^{2}-4 D q^{4}+p^{2}+O\left(q^{5}, p q^{3}, p^{2} q, p^{3}\right) .
$$

For $D<0$ and $\delta \geq 0$ this derivative is positive in the domain $U_{c} \backslash\{0\}$. On any trajectory, starting in the domain $U_{c} \cap\{V>0\}$ the derivative $d V / d t$ is greater than a positive constant, depending on the initial condition. Therefore any such trajectory leaves $U_{c}$.

\subsection{Example: stability loss in billiard dynamics}

Consider a particle moving in a domain, bounded on $\mathbb{R}^{2}=\{(x, y)\}$ by the curves

$$
y=1 \quad \text { and } \quad y=d_{2} x^{2}+d_{4} x^{4}+O\left(x^{5}\right) .
$$

By definition the motion takes place with a constant velocity until a collision with the boundary. The collision is elastic: the angle of incidence equals the angle of reflection, see Fig. 5

There exists a periodic solution $\gamma$ such that the particle moves along the $y$-axis. This is a so-called two-link billiard trajectory. Linear stability of $\gamma$ is determined by the length of the link $l=1$ and by the curvatures $k_{1}=0, k_{2}=2 d_{2}$ of the boundary at the collision points. More precisely (see for example, [41]), multipliers of a two-link billiard trajectory satisfy the equation

$$
\lambda^{2}-\left(2-8 d_{2}\right) \lambda+1=0 .
$$

Therefore, $\gamma$ is elliptic if $0<d_{2}<1 / 2$ and hyperbolic if $d_{2}<0$ or $d_{2}>1 / 2$. We consider the loss of stability when $d_{2}$ increases and passes through $1 / 2$. In this case the multipliers collide at the point -1 and leave the unit circle drifting away along the real axis. 
Proposition 2.1 The stability loss is soft if $d_{4}<1 / 8$ and hard if $d_{4}>1 / 8$.

Remark 2.2 It is possible to guess that the value $d_{2}=1 / 8$ corresponds to the change of the two types of the stability loss by using the following argument. The equation $d_{4}=1 / 8$ is fulfilled, for example, for the circle with center at the point $(x, y)=(0,1)$. In this case $\gamma$ belongs to a one-parameter family of two-link periodic orbits of the billiard system. We do not see anything like this in Fig 4. Therefore one should expect that $d_{4}=1 / 8$ is a boundary value of the parameter $d_{4}$ between the hard and soft stability loss.

Proof of Proposition 2.1. It is convenient to study the dynamics in terms of the billiard map. This map can be defined in several ways. Here we prefer the version which regards the billiard system as a discrete Lagrangian system. It is well-known that billiard trajectories are extremals of the length (the Fermat principle). More precisely, if $p_{1}, p_{2}, p_{3}$ are three successive collision points of a billiard trajectory then for fixed $p_{1}$ and $p_{3}$ the point $p_{2}$ should make the Euclidean length $l\left(p_{1}, p_{2}\right)+l\left(p_{2}, p_{3}\right)$ extremal. We parameterize the billiard boundary by the coordinate $x$. Then assuming that $p^{\prime}=\left(x^{\prime}, y^{\prime}\right)$ lies on the first curve $(2.5)$ while $p^{\prime \prime}=\left(x^{\prime \prime}, y^{\prime \prime}\right)$ lyes on the second one, we have:

$$
\begin{aligned}
l\left(p^{\prime}, p^{\prime \prime}\right)= & l\left(x^{\prime}, x^{\prime \prime}\right)=\sqrt{\left(x^{\prime}-x^{\prime \prime}\right)^{2}+\left(1-d_{2}\left(x^{\prime \prime}\right)^{2}-d_{4}\left(x^{\prime \prime}\right)^{4}\right)^{2}+O_{5}\left(x^{\prime}, x^{\prime \prime}\right)} \\
= & 1+\frac{\left(x^{\prime}-x^{\prime \prime}\right)^{2}}{2}-d_{2}\left(x^{\prime \prime}\right)^{2} \\
& -\frac{\left(x^{\prime}-x^{\prime \prime}\right)^{4}}{8}+\frac{d_{2}}{2}\left(x^{\prime}-x^{\prime \prime}\right)^{2}\left(x^{\prime \prime}\right)^{2}-d_{4}\left(x^{\prime \prime}\right)^{4}+O_{6}\left(x^{\prime}, x^{\prime \prime}\right) .
\end{aligned}
$$

Let $p_{1}, p_{2}, p_{3}, p_{4}$ be 4 successive collision points of a billiard trajectory: $p_{1}, p_{3}$ lie on the first curve (2.5) and $p_{2}, p_{4}$ on the second one. Consider the billiard map

$$
\left(p_{1}, p_{2}\right) \mapsto b\left(p_{1}, p_{2}\right)=\left(p_{3}, p_{4}\right), \quad p_{j}=\left(x_{j}, y_{j}\right)
$$

The periodic trajectory $\gamma$ corresponds to the fixed point $p_{1}=p_{3}=(0,1), p_{2}=p_{4}=(0,0)$ of $b$. In the case $d_{2}=1 / 2$ the equations

$$
\frac{\partial}{\partial x_{2}}\left(l\left(x_{1}, x_{2}\right)+l\left(x_{3}, x_{2}\right)\right)=0, \quad \frac{\partial}{\partial x_{3}}\left(l\left(x_{3}, x_{2}\right)+l\left(x_{3}, x_{4}\right)\right)=0 .
$$

take the form (up to terms of order 5 in $x_{1}$ and $x_{2}$ )

$$
x_{3}=-x_{1}-2 x_{1}^{2} x_{2}+\left(1-8 d_{4}\right) x_{2}^{3}, \quad x_{4}=-2 x_{1}-x_{2}+2 x_{1}^{3}+2 x_{1} x_{2}^{2}+2\left(1-8 d_{4}\right) x_{2}^{3} .
$$

The final argument is somewhat implicit. The formal integral (a constant of motion):

$$
x_{1}^{2}\left(1+\left(x_{1}-x_{2}\right)^{2}\right)+\left(1-8 d_{4}\right) x_{2}^{2}\left(x_{1}-\frac{x_{2}}{2}\right)^{2}+O_{6}\left(x_{1}, x_{2}\right)
$$

is (up to small terms of order 6 ) positive near the origin if $d_{4}<1 / 8$ and sign-indefinite if $d_{4}>1 / 8$. This implies (formal) stability for $d_{4}<1 / 8$ and instability for $d_{4}>1 / 8$. This proves the proposition. 


\section{Hamiltonian Poincaré-Andronov-Hopf bifurcation}

\subsection{Theorem on the stability loss}

Consider a Hamiltonian system with 2 degrees of freedom (i.e., $\operatorname{dim} \mathcal{X}=4$ ) and a family of equilibrium positions $x_{\kappa}, \kappa \in\left(-\kappa_{0}, \kappa_{0}\right)$. We assume that for negative $\kappa$ eigenvalues of $A_{\kappa}$ (see (1.2)) are pairwise distinct and lie on the imaginary axis $i \mathbb{R}$ while for positive $\kappa$ they have the form $\pm \alpha \pm i \beta, \alpha, \beta \in \mathbb{R} \backslash\{0\}$. Hence, when $\kappa$ increases and passes through zero, a loss of stability of $x_{\kappa}$ takes place because two pairs of eigenvalues collide on the imaginary axis and drift away in the horizontal direction. This type of the stability loss is known as the Hamiltonian Poincaré-Andronov-Hopf bifurcation. ${ }^{5}$

The characteristic polynomial at the point $x_{\kappa}$ is even. Let $D=D(\kappa)$ be discriminant of the corresponding quadratic polynomial. We assume that $d D /\left.d \kappa\right|_{\kappa=0} \neq 0$. Then according to [6] there exist local coordinates $p=\left(p_{1}, p_{2}\right)$ and $q=\left(q_{1}, q_{2}\right)$ on $\mathcal{X}$ such that

(a) $p$ and $q$ depend smoothly on $\kappa$,

(b) the symplectic structure on $\mathcal{X}$ equals $d p_{1} \wedge d q_{1}+d p_{2} \wedge d q_{2}$,

(c) the points $x_{\kappa}$ have zero coordinates,

(d) the Hamiltonian function equals

$$
H_{\kappa}=H_{*}+\kappa O\left(\rho^{4}\right)+O\left(\rho^{5}\right), \quad H_{*}=\omega(\kappa) I+G,
$$

where

$$
\begin{gathered}
I=q_{1} p_{2}-q_{2} p_{1}, \quad G=\frac{1}{2} p^{2}-\frac{\varepsilon(\kappa)}{2} q^{2}+q^{2}\left(A q^{2}+B I+C p^{2}\right), \\
\frac{\varepsilon(\kappa)}{\kappa} \geq 0, \quad q^{2}=q_{1}^{2}+q_{2}^{2}, \quad p^{2}=p_{1}^{2}+p_{2}^{2}, \quad \rho^{2}=q^{2}+p^{2} .
\end{gathered}
$$

We define the balls

$$
U_{r}(\kappa)=\{(q(\kappa), p(\kappa)): \rho(\kappa)<r\} .
$$

Theorem 2 [78]. Suppose $d \varepsilon /\left.d \kappa\right|_{\kappa=0} \neq 0$. Then there are two possibilities.

1. Suppose $A>0$. Then for $\kappa$ passing through zero a soft stability loss takes place: there exists a constant $c>0$ such that for small $r \geq 0$ any solution with initial data in $U_{r}(\kappa)$ stays in $U_{c \sqrt{\kappa+r}}(\kappa)$.

2. Suppose $A<0$. Then for $\kappa$ passing through zero a hard stability loss takes place: for any $\kappa \in\left[0, \kappa_{0}\right)$ and any small $r>0$ the domain $U_{r}(\kappa)$ contains initial conditions such that the corresponding solution leaves $U_{c}(\kappa)$, where $c>0$ does not depend on $r$ and $\kappa$.

\subsection{Proof of Theorem 2}

System determined by the Hamiltonian function $H_{*}$ is completely integrable because the Poisson bracket $\{I, G\}=0$. For any $\kappa \in\left(-\kappa_{0}, \kappa_{0}\right)$ we put

$$
\begin{aligned}
& Q_{a, h}=\left\{(q, p) \in \mathbb{R}^{4}: I=a, H_{*}=h\right\}, \\
& m^{2}=\min _{Q_{a, h}} \rho^{2}, \quad M^{2}=\max _{Q_{a, h}} \rho^{2} .
\end{aligned}
$$

\footnotetext{
${ }^{5} \mathrm{~A}$ necessary condition for the such a bifurcation is the existence of two nontrivial Jordan blocks in the Jordan form of the matrix $A_{0}$.
} 
The following technical proposition says that Theorem 2 holds if we replace the Hamiltonian function by its integrable truncation $H_{*}$.

Proposition 3.1 Suppose $\varepsilon>0, I$ and $h$ are small constants, Then for some constant $b=b(A, B, C)$

$$
M^{2} \leq b(\varepsilon+m)
$$

Here is a sketch of the proof. The conditional extrema $m$ and $M$ are achieved at points where the covectors $\operatorname{grad} I, \operatorname{grad} G$, and $\operatorname{grad} \rho^{2}$ are linearly dependent. This is equivalent to the inequality

$$
\begin{gathered}
\operatorname{rank}\left(\begin{array}{cccc}
p_{2} & -p_{1} & -q_{2} & q_{1} \\
q_{1} U & q_{2} U & p_{1} V & p_{2} V \\
q_{1} & q_{2} & p_{1} & p_{2}
\end{array}\right) \leq 2, \\
U=-\varepsilon+4 A q^{2}+2 B I+2 C p^{2}, \quad V=1+2 C q^{2} .
\end{gathered}
$$

The quantity $V$ is of order 1 while $U=O\left(\kappa, p^{2}+q^{2}\right)$ is small. Therefore $V \neq U$ and condition (3.3) is equivalent to

$$
q_{1} p_{1}+q_{2} p_{2}=0 \text {. }
$$

The functions $I, G$, and $\rho^{2}$ as well as the left-hand side of (3.4) do not change after transformations $q \mapsto R q, p \mapsto R p$, where $R$ is a rotation in the plane $\mathbb{R}^{2}$. Hence we may assume that

$$
q=(\sqrt{\xi}, 0), \quad p=(0, \sigma \sqrt{\eta}), \quad \sigma=\operatorname{sign} c .
$$

The functions $I, G, \rho^{2}$ take the form

$$
I=\sigma \sqrt{\xi \eta}, \quad G=\frac{1}{2} \eta-\frac{\varepsilon}{2}+\xi(A \xi+B I)+C I^{2}, \quad \rho^{2}=\xi+\eta .
$$

Putting $\delta=h-C a^{2}$, we obtain the equations

$$
\xi \eta=a^{2}, \quad \frac{1}{2} \eta-\left(\frac{\varepsilon}{2}-B a\right) \xi+A \xi^{2}=\delta
$$

Hence

$$
m=m(a, \delta)=\xi_{m}+\eta_{m}, \quad M=M(a, \delta)=\xi_{M}+\eta_{M},
$$

where $\left(\xi_{m}, \eta_{m}\right)$ and $\left(\xi_{M}, \eta_{M}\right)$ are nonnegative solutions of equations (3.5). In particular, $\xi_{m} \leq \xi_{M}$ are nonnegative roots of the polynomial

$$
\mathcal{P}(\xi)=A \xi^{3}-\left(\frac{\varepsilon}{2}-B a\right) \xi^{2}-\delta \xi+A a^{2} / 2
$$

We skip final technical argument which implies (3.2).

If $\rho$ and $\kappa$ are small, the term $\kappa O\left(\rho^{4}\right)+O\left(\rho^{5}\right)$ in (3.1) can be regarded as a small perturbation of the function $H_{*}$. According to KAM-theory Diophantine two-dimensional invariant tori of the unperturbed system survive the perturbation. Being hypersurfaces on the energy levels $H_{\kappa}=$ const, they prevent trajectories, starting in $U_{r}(\kappa)$ to leave 
$U_{c \sqrt{\kappa+r}}(\kappa)$, where $c$ is any constant larger than $b$, defined in from Proposition 3.1. This finishes proof of Theorem 2 in the case $A>0$.

In the case $A<0$ consider the function $V=p_{1} q_{1}+p_{2} q_{2}$. We have:

$$
\frac{d V}{d t}=4 p^{2}+2 q^{2}\left(-2 A q^{2}+\varepsilon+B I+2 C P^{2}\right)+\kappa O\left(\rho^{4}\right)+O\left(\rho^{5}\right) .
$$

Therefore $d V / d t>0$ for $A<0$ and small $\kappa \geq 0$ in the domain

$$
U_{\kappa, r}^{+}=U_{\kappa, r} \cap\{V>0\} \cap\{I>0\} .
$$

On any solution with initial condition in $U_{\kappa, r}^{+}$the function $V$ increases until the solution leaves $U_{\kappa, r}$.

\subsection{Generalizations and remarks}

1. In the case $\kappa=0, A>0$ formal stability ${ }^{6}$ of $x_{0}$ was proven in [72] while its Liapunov stability was established in [39]. A modern view on the stability problem in the case $\kappa=0$ is given in [44].

2. Analogously to Remark 2.1 we expect that in the case $A<0$ and $\kappa>0$ almost all solutions starting in $U_{r}(\kappa)$ leave $U_{c}(\kappa)$.

3. Theorem 2 remains valid in the case of more than two degrees of freedom provided additional eigenvalues of the matrix $A_{\kappa}$ are purely imaginary and at the point $\kappa=0$ there are no resonances of order 4 or less between the eigenvalues. In this case the words "Lyapunov stability" should be replaced by the words "metric stability" or "stability for majority initial conditions" because of possible presence of the Arnold diffusion.

4. Consider the Hamiltonian Poincaré-Andronov-Hopf bifurcation for a periodic solution in the simplest possible case of two and a half degrees of freedom. More precisely, consider a Hamiltonian system with 2 degrees of freedom, where the Hamiltonian function $H_{\kappa}$ depends periodically on time. Let $\gamma_{\kappa}$ be a smooth family of nondegenerate periodic trajectories. Suppose multipliers of $\gamma_{\kappa}$ lie on the unit circle $S^{1} \subset \mathbb{C}$ for $\kappa \leq 0$, when $\kappa=0$ two pairs of multipliers collide at two distinct complex conjugate points and for $\kappa>0$ take the form

$$
\lambda_{\kappa}, \bar{\lambda}_{\kappa}, \lambda_{\kappa}^{-1}, \text { and } \bar{\lambda}_{\kappa}^{-1} \text { with } \lambda_{\kappa} \in \mathbb{C} \backslash \mathbb{R}, \quad\left|\lambda_{\kappa}\right| \neq 1 .
$$

It is known, [15] that the normal form of the corresponding Hamiltonian $H_{\kappa}$ near $\gamma_{\kappa}$ coincides with (3.1) up to a constant multiplier, but the term $\kappa O\left(\rho^{4}\right)+O\left(\rho^{5}\right)$ in general periodically depends on time. Arguments, essentially the same as in Section 3.2, prove a version of Theorem 2 in which the equilibrium positions $x_{\kappa}$ are replaced by periodic trajectories $\gamma_{\kappa}$ and Lyapunov stability by metric orbital stability.

5. Multidimensional extension of the result from item 4 is straightforward provided multipliers of $\gamma_{0}$ do not satisfy resonances of orders 4 or less. In the autonomous case (3 or more degrees of freedom) as the parameter $\kappa$ the value of the energy may be chosen.

\footnotetext{
${ }^{6}$ i.e., stability of the system, determined by any truncation of the (in general, diverging) normal form
} 


\subsection{Example: libration points in RTBP}

Recall that the restricted three-body problem (RTBP) is a Hamiltonian system which determines the motion of a particle $A$ (Asteroid) in the gravity force field of two heavy particles $S$ and $J$ (Sun and Jupiter). Mass of Asteroid is assumed to be negligibly small w.r.t. the masses $m_{S}$ and $m_{J}$ of Sun and Jupiter. Hence, motion of the latter ones is determined by the two-body problem. W.l.g. their mass center is fixed. We consider planar motions of this three-body system. Traditionally it is assumed that $m_{s}+m_{J}=1$.

First, suppose that orbits of the two large bodies are circular. In this case the distance between Sun and Jupiter equals constant. Then the coordinate frame with the origin at the mass center and the first axis through the Sun and Jupiter rotates uniformly about the origin. The problem of the Asteroid motion in this plane has 5 equilibrium positions. We are interested in $L_{4}$ and $L_{5}$, the Lagrange triangular libration points points situated at the vertices of equilateral triangle with other vertices at the points $S$ and $J$.

These equilibrium positions are linearly stable provided

$$
0<m_{J} \leq m_{*}=0.03852 \ldots, \quad m_{*}\left(1-m_{*}\right)=1 / 27 .
$$

Moreover, for all values of $m_{J}$ satisfying (3.7) except two special ones $L_{4}$ and $L_{5}$ are Lyapunov stable, [48], see also [6]. For these two exceptional values of $m_{J}$ resonances of orders 3 and 4 occur and generate instability of the libration points, [48]. We are interested in the Hamiltonian-Andronov-Hopf bifurcation which appears when $m_{J}$ increases and passes through the value $m_{*}$. We put $\kappa=m_{J}-m_{*}$.

The characteristic polynomial equals $\lambda^{4}+\lambda^{2}+27 m_{j}\left(1-m_{J}\right) / 4$. Hence, $d D /\left.d \kappa\right|_{\kappa=-27\left(1-2 m_{*}\right)}<$ 0 . The value $A=0.603 \ldots>0$ was computed in [73]. Therefore loss of stability in this case is soft.

The same conclusion remains valid in the elliptic planar restricted three-body problem in the case of a small eccentricity $e$ in the Sun-Jupiter system. In this case the triangular libration points are replaced by triangular periodic solutions. Because of smallness of $e$ values of the parameters $m_{*}, d D /\left.d \kappa\right|_{\kappa=0}$, and $A$ are close to that from the circular problem. Therefore when $m_{J}$ passes through the bifurcational value a soft stability loss takes place. A stability diagram on the rectangle $\left\{\left(m_{J}, e\right): 0 \leq m_{J} \leq 1 / 2,0 \leq e \leq 1\right\}$ is presented in [47].

\section{Stability loss delay as an unavoidable phenomenon in analytic systems}

Starting from this section we study stability loss in the context of dynamical bifurcations. We consider slow-fast ODEs systems (1.4) and slow-fast maps (1.5). We use notation and terminology introduced in Section 1.

In what follows $C, c, C_{i}, c_{i}$ are positive constants which should be taken large enough.

Theorem 3 [53] Assume that the functions $f, g$ in (1.4) can be analytically continued with respect to $x, \kappa$ into an $\varepsilon$-independent complex neighbourhood of the state $L_{K\left(\tau_{*}\right)}$ and remain smooth in $\varepsilon$. Then the phase point $x(t), \kappa(t)$ whose motion starts at $\tau=\tau_{0}, \kappa=\kappa_{0}$ 
in $C_{1}^{-1}$-neighbourhood of the state $L_{\kappa_{0}}$, moves in $C_{3}^{-1} \varepsilon$-neighbourhood of the state $L_{K(\tau)}$ for $\tau_{0}+C_{4} \varepsilon|\ln \varepsilon| \leq \tau \leq \tau_{*}+C_{2}^{-1}$.

The part of this theorem concerning the motion for $\tau_{0}+C_{4} \varepsilon|\ln \varepsilon| \leq \tau \leq \tau_{*}-C_{5}^{-1}$ is a statement from [85] (for the case of an equilibrium) and [63] (for the case of a periodic trajectory).

Theorem 4 [53] Assume that functions $f, g$ in (1.5) can be analytically continued with respect to $x, \kappa$ into an $\varepsilon$-independent neighbourhood of the state $L_{K\left(\tau_{*}\right)}$ and remain smooth in $\varepsilon$. Let the point $x_{0}, \kappa_{0}$ belong to $C_{1}^{-1}$-neighbourhood of the state $L_{\kappa_{0}}$. Then $n$-th iteration of the map (1.5) with the initial condition $x_{0}, \kappa_{0}$ belongs to $C_{3}^{-1} \varepsilon$-neighbourhood of the state $L_{K(\varepsilon n)}$ for $\tau_{0}+C_{4} \varepsilon|\ln \varepsilon| \leq \varepsilon n \leq \tau_{*}+C_{2}^{-1}$.

Note that states $L_{\kappa}, \kappa \in \mathcal{K}$ form an invariant manifold of system (1.4) (or map (1.5)) for $\varepsilon=0$. Theorems 3,4 are dealing with the case when this manifold is not normally hyperbolic. Thus, the Fenichel theorem [27] does not guarantee its persistence for $\varepsilon>0$.

Theorem 3 is proven in [53]. The proof of Theorem 4 is completely similar (details are presented in [28], see the proof of Theorem 5 there). Further results on stability loss delay for slow-fast maps are contained in [29, 30]. Here we explain the scheme of the proof of Theorem 3 for the case when the state $L_{\kappa}$ is an equilibrium of the fast system.

\subsection{Sketch of the proof of Theorem 3 when $L_{\kappa}$ is an equilibrium}

Let the state $L_{\kappa}$ be determined by the equation $x=X(\kappa)$. Our aim is to introduce instead of $x$ a new fast variable $\xi$ such that $\{\xi=0\}$ is an invariant surface of system (1.4) up to $O\left(\exp \left(-C^{-1} / \varepsilon\right)\right)$, where $C$ is a positive constant. More exactly, we would like to reduce the fast equation to the form

$$
\dot{\xi}=A(\kappa) \xi+O\left(\varepsilon|\xi|+|\xi|^{2}\right)+h(\kappa, \varepsilon), \quad h=O\left(\exp \left(-C^{-1} / \varepsilon\right)\right)
$$

Here $A(\kappa)=(\partial f(x, \kappa, 0) / \partial x))_{x=X(\kappa)}$. The required transformation of variables can be constructed, e.g., as a composition of many, $\sim 1 / \varepsilon$, transformations. After $j$ transformations the fast equation takes the form

$$
\dot{\xi}_{j}=A(\kappa) \xi_{j}+O\left(\varepsilon\left|\xi_{j}\right|+\left|\xi_{j}\right|^{2}\right)+h_{j}(\kappa, \varepsilon)
$$

with the fast variable $\xi_{j}$. For the first of these transformations $\xi_{1}=x-X(\kappa)$ and $h_{1}=O(\varepsilon)$. The variable $\xi_{j+1}$ is introduced by the equation $\xi_{j+1}=\xi_{j}+A^{-1}(\kappa) h_{j}(\kappa, \varepsilon)$ (note that $A(\kappa)$ is a non-degenerate matrix). Analyticity implies that norms of $h_{j}$ decay with $j$ at least as a geometric series with the common ratio which does not depend on $\varepsilon$ in some neighbourhood of the bifurcation point $\left(X\left(K\left(\tau_{*}\right)\right), K\left(\tau_{*}\right)\right)$. One can take this common ratio equal to $1 / 2$ for the number of iterations not exceeding $c^{-1} / \varepsilon$. Estimates here are similar to those in $[58,51]$. After $\sim c^{-1} / \varepsilon$ iterations we obtain equation (4.1) with the exponentially small term $h$. Note that $x=X(\kappa)+\xi+O(\varepsilon)$.

Now consider a phase point $x(t), \kappa(t)$ of system (1.4) whose motion starts at $\tau=$ $\tau_{0}, \kappa=\kappa_{0}$ in $C_{1}^{-1}$-neighbourhood of the state $L_{\kappa_{0}}$. During the slow time $O(\varepsilon \ln \varepsilon)$ this 
phase point is attracted into $O(\varepsilon)$-neighbourhood of the point $(X(K(\varepsilon t)), K(\varepsilon t))$ [85]. It continues to move into $O(\varepsilon)$-neighbourhood of $(X(K(\varepsilon t)), K(\varepsilon t))$ at least up to arrival into a neighbourhood of the bifurcation point $\left(X\left(K\left(\tau_{*}\right)\right), K\left(\tau_{*}\right)\right)$ where variables $\xi, \kappa$ and equation (4.1) can be used for the description of motion. In the new variables the phase point is $\xi(t), \kappa(t)$. Real parts of eigenvalues of the matrix $A(K(\tau))$ are negative for $\tau_{0} \leq \tau<\tau_{*}$. Therefore the point $\xi(t)$ is attracted into an $O\left(\exp \left(-C_{2}^{-1} / \varepsilon\right)\right)$-neighbourhood of the origin, $\xi=0$, and remains in this neighbourhood at least while $\tau<\tau_{*}-\mu(\tau)$, where $\mu(\tau) \rightarrow 0$ as $\tau \rightarrow \tau_{*}$. After this, at least time of order $1 / \varepsilon$ is needed to reach values $|\xi(t)| \sim \varepsilon$.

Thus $|\xi(t)|=O(\varepsilon)$ for $\tau_{*}-\mu(\tau) \leq \tau \leq \tau_{*}+C_{2}^{-1}$ and $|x(t)-X(\kappa(\varepsilon t))|=O(\varepsilon)$ for $\tau_{0}+C_{4} \varepsilon|\ln \varepsilon| \leq \tau \leq \tau_{*}+C_{2}^{-1}$. During this time $|\kappa(\varepsilon t)-K(\varepsilon t)|=O(\varepsilon)$, and thus $\mid x(t)-$ $X(K(\varepsilon t)) \mid=O(\varepsilon)$. This is the statement of Theorem 3 for the case of an equilibrium.

We have constructed the reduction of equation of motion to the form (4.1) via series of $\sim 1 / \varepsilon$ shifts of the coordinate origin. Instead, one can find the required shift in the form of a polynomial in $\varepsilon$ :

$$
x=\xi+X(\kappa)+\varepsilon u_{N}(\kappa, \varepsilon), \varepsilon u_{N}(\kappa, \varepsilon)=a_{1}(\kappa) \varepsilon+a_{2}(\kappa) \varepsilon^{2}+\ldots+a_{N}(\kappa) \varepsilon^{N} .
$$

Coefficients $a_{j}$ can be determined recursively from the condition that the term of order $\varepsilon^{j}$ in $\dot{\xi}$ vanishes at $\xi=0$. At each step of this recursive procedure one should differentiate terms obtained earlier. For analytic functions, norm of $j$ th derivative is bounded by $c^{j} j$ !. This allows to show that $a_{j} \lesssim c^{j} j ! \sim\left(\frac{c j}{e}\right)^{j+1 / 2}$ (estimates here are similar to those in [67]). Thus, an estimate for $\varepsilon^{j} a_{j}$ grows with $j$ for $j>j_{*}=e /(c \varepsilon)$. Take $N$ equal to the integer part of $1 /(c \varepsilon)$. This gives $u_{N}(\kappa, \varepsilon) \lesssim 1$ and exponentially small $h$ in (4.1). Such truncation of series is a standard approach in asymptotic analysis (cf. [21]).

The statement about the existence of stability loss delay in [8] is based on the assumption that it is possible to continue the expansion in (4.2) to get a convergent series. If this assumption is satisfied, then the situation is reduced to that in the case of trivial stability loss delay. However, this is a highly degenerate situation. In general there is no reason to expect such a convergence.

Example (A simplified Pontryagin-Shishkova example [69]) Consider the system

$$
\dot{z}=(\kappa-i)(z-\kappa), \quad \dot{\kappa}=\varepsilon, \quad z=x_{1}+i x_{2} \in \mathbb{C} \text {. }
$$

This is a slow-fast system with two real fast variables $x_{1}, x_{2}$ and one slow $\kappa$ variable. The fast equation has an equilibrium at $z=\kappa$. We are looking for an asymptotic series

$$
z=\kappa+\varepsilon u(\kappa, \varepsilon), \quad \varepsilon u(\kappa, \varepsilon)=a_{1}(\kappa) \varepsilon+a_{2}(\kappa) \varepsilon^{2}+\ldots
$$

which is a solution of (4.3). Plug (4.4) into (4.3) and equate terms with the same degree in $\varepsilon$. We get

$$
a_{1}=\frac{1}{\kappa-i}, \quad a_{j+1}=\frac{1}{\kappa-i} \frac{d a_{j}}{d \kappa}, \quad j=1,2, \ldots
$$


Thus

$$
a_{j}(\kappa)=\frac{(-1)^{j+1}(2 j-3) ! !}{(\kappa-i)^{2 j-1}}, \quad j=2,3, \ldots
$$

The series in (4.4) diverges for any $\kappa$ and $\varepsilon \neq 0$.

One should not expect a stability loss delay in typical non-analytic systems. If the system is of smoothness $\mathcal{C}^{l}$ only, then the procedure of the recursive construction of the polynomial (4.2) stops after $l$ steps. Thus, one can take $N=l-1$ in (4.2) and $h=O\left(\varepsilon^{l}\right)$ in (4.1). This does not imply the stability loss delay: if $|\xi(t)| \sim \varepsilon^{l}$ at $\tau=\tau_{*}$, then it typically reaches a value $\sim 1$ by a moment of slow time $\tau_{d}>\tau_{*}$ such that $\tau_{d} \rightarrow \tau_{*}$ as $\varepsilon \rightarrow 0$. In a typical case, when real part of complex conjugate eigenvalues, responsible for stability loss, grows linearly with $\tau-\tau_{*}$, we get $\tau_{d}=\tau_{*}+O(\sqrt{l \varepsilon|\ln \varepsilon|})$. An example can be found in [53].

If the system is of smoothness $\mathcal{C}^{\infty}$, then the above recursive procedure constructs the polynomial (4.2) for any $N$. For functions in the Gevrey class $G^{\sigma}, \sigma>1$, the norm of $j$ th derivative is bounded by $c^{j}(j !)^{\sigma}$. In this case $a_{j} \lesssim c_{1}^{j}(j !)^{\sigma} \sim\left(\frac{c_{2} j}{e}\right)^{\sigma(j+1 / 2)}$ (estimates here are similar to those in [67]). The optimal truncation requires $N \sim C^{-1} / \varepsilon^{1 / \sigma}$. Hence $h=O\left(\exp \left(-c^{-1} / \varepsilon^{1 / \sigma}\right)\right)$ in (4.1). Again, this does not imply the stability loss delay: if $|\xi(t)| \sim \exp \left(-c^{-1} / \varepsilon^{1 / \sigma}\right)$ at $\tau=\tau_{*}$, then typically it reaches a value $\sim 1$ by a moment of slow time $\tau_{d}=\tau_{*}+O\left(\varepsilon^{(1-1 / \sigma) / 2}\right)$. In [53] there is an example of absence of stability loss delay in a system of smoothness $\mathcal{C}^{\infty}$.

As the phenomenon of stability loss is associated with the analyticity, it can be destroyed by an exponentially small non-analytic perturbation. In particular, it can be destroyed by an exponentially small noise. Effect of noise on stability loss delay is considered in $[9,77]$.

A remarkable aspect of stability loss delay phenomenon is the existence of a maximal delay (called also a buffer point or, in French, butée) [20]. Let us describe the maximal delay effect in a simplest situation of the stability loss of an equilibrium, assuming that there are two fast variables and one slow variable. The slow variable is supposed to be the slow time $\kappa=\varepsilon t$ and equations of motion have the form

$$
\dot{x}=f(x, \kappa), \quad \dot{\kappa}=\varepsilon, \quad x \in \mathbb{R}^{2}, \quad \kappa \in \mathbb{R}^{1} .
$$

We assume that the fast system has an equilibrium at $x=X(\kappa), \kappa \in(-\infty, \infty)$, that this equilibrium depends smoothly on $\kappa$, and that real parts of its eigenvalues are negative for $\kappa<\kappa_{*}$ and positive for $\kappa>\kappa_{*}$. Denote

$$
\mathcal{L}=\{(\kappa, x): x=X(\kappa), \kappa \in(-\infty, \infty)\}
$$

The function $f$ in (4.5) is supposed to be analytic in a complex neighbourhood of $\mathcal{L}$.

If some mild assumptions are satisfied, then system (4.5) has solutions $x=X_{ \pm}(\varepsilon t), t \in$ $(-\infty, \infty)$ such that $\left|X_{-}(\varepsilon t)-X(\varepsilon t)\right|=O(\varepsilon)$ in the limit $t \rightarrow-\infty$, and $\left|X_{+}(\varepsilon t)-X(\varepsilon t)\right|=$ $O(\varepsilon)$ in the limit $t \rightarrow+\infty$. Denote

$$
\mathcal{L}_{ \pm}=\left\{(\kappa, x): x=X_{ \pm}(\kappa), \kappa \in(-\infty, \infty)\right\}
$$




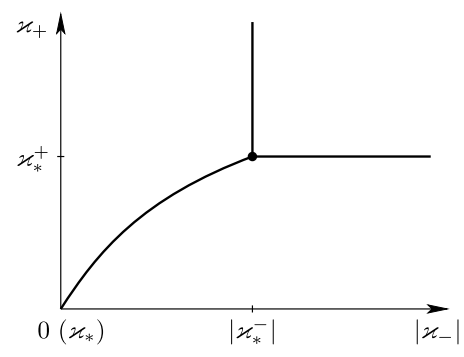

Figure 6: In-out (entrance-exit) function

Typically, $\mathcal{L}_{-}$and $\mathcal{L}_{+}$do not coincide. Their mismatch at $\kappa_{*}$ is an exponentially small value, $X_{+}\left(\kappa_{*}\right)-X_{-}\left(\kappa_{*}\right) \sim \exp \left(-C_{2}^{-1} / \varepsilon\right)$. It is a manifestation of stability loss delay phenomenon that these two solutions stay close to each other on some interval of a length $\sim 1$ of values of $\kappa=\varepsilon t$ around $\kappa_{*}$. Namely, there are values $\kappa_{*}^{ \pm}$such that $\kappa_{*} \in\left(\kappa_{*}^{-}, \kappa_{*}^{+}\right)$ and

- $X_{+}(\kappa)-X_{-}(\kappa)=O\left(\exp \left(-c^{-1} / \varepsilon\right)\right)$ for $\kappa \in\left(\kappa_{*}^{-}+c^{-1}, \kappa_{*}^{+}-c^{-1}\right)$,

- $X_{+}(\kappa)-X_{-}(\kappa)=O(\varepsilon)$ for $\kappa \in\left(\kappa_{*}^{-}+c \varepsilon|\ln \varepsilon|, \kappa_{*}^{-}+c^{-1}\right) \cup\left(\kappa_{*}^{+}-c^{-1}, \kappa_{*}^{+}-c \varepsilon|\ln \varepsilon|\right)$,

- $\left|X_{+}(\kappa)-X_{-}(\kappa)\right|>c^{-1}$ for $\kappa \in \mathbb{R} \backslash\left(\kappa_{*}^{-}-c \varepsilon|\ln \varepsilon|, \kappa_{*}^{+}+c \varepsilon|\ln \varepsilon|\right)$.

Note that $X_{-}(\kappa)$ and $X_{+}(\kappa)$ are $O(\varepsilon)$-close to $X(\kappa)$ at $\kappa \in\left(-\infty, \kappa_{*}^{+}-c \varepsilon|\ln \varepsilon|\right)$ and $\kappa \in\left(\kappa_{*}^{-}+c \varepsilon|\ln \varepsilon|, \infty\right)$, respectively. We can say that the phase point $X_{-}(\kappa)$ is attracted to $\mathcal{L}_{+}$near $\kappa=\kappa_{*}^{-}$and is repelled from $\mathcal{L}_{+}$near $\kappa=\kappa_{*}^{+}$.

Now consider a phase point which is attracted to $\mathcal{L}$ (and thus to $\mathcal{L}_{-}$) near some $\kappa=\kappa_{-}<\kappa_{*}^{-}$. In the stability domain it moves near $\mathcal{L}_{-}$longer than $X_{+}(\kappa)$, and thus is attracted closer to $\mathcal{L}_{-}$than $X_{+}(\kappa)$. As a result, in the instability domain it stays closer to $\mathcal{L}_{-}$than to $\mathcal{L}_{+}$, and is repelled from $\mathcal{L}_{+}$(and thus from $\mathcal{L}$ ) together with $X_{-}(\kappa)$, near $\kappa=\kappa_{*}^{+}$. No delay beyond the point $\kappa_{+}+c \varepsilon|\ln \varepsilon|$ is possible. Note that all phase points attracted to $\mathcal{L}$ before $\kappa=\kappa_{*}^{-}$are repelled from $\mathcal{L}$ about simultaneously (on slow time $\kappa$ scale) near $\kappa=\kappa_{*}^{+}$. Phase points, that are attracted to $\mathcal{L}$ near $\kappa=\kappa_{-}>\kappa_{*}^{-}$, are repelled from $\mathcal{L}$ near some $\kappa=\kappa_{+}<\kappa_{*}^{+}$.

Attraction to $\mathcal{L}$ and repelling from it are instant events in the slow time $\kappa$ scale in the limit $\varepsilon \rightarrow 0$. One can define an in-out or entrance-exit function $\Pi(\cdot)$ such that for the time of attraction $\kappa_{-}$the time of repelling is $\kappa_{+}=\Pi\left(\kappa_{-}\right)$. A typical graph of this function is shown in Fig. 6 (for $\kappa_{*}=0$ ). The manifestation of the maximal delay effect is that the value of this function equals the constant $\kappa_{*}^{+}$for $\kappa<\kappa_{-}$. Calculation of the in-out function can be performed via study behaviour of solutions in complex time.

In the case of a trivial delay $\mathcal{L}_{-}$and $\mathcal{L}_{+}$coincide, and there is no maximal delay phenomenon.

\section{$5 \quad$ Stability loss delay time for an equilibrium}

In this section we consider system (1.4) in the case when the state $L_{\kappa}$ is an equilibrium $x=X(\kappa)$ of the fast system. Thus, $G(\kappa)=g(X(\kappa), \kappa, 0)$ in the slow equation (1.6). We 
will calculate an asymptotic formula for the delay time in the case when this time is less than the maximal delay introduced at the end of the previous section. This is relatively well developed subject. We follow [54] in the exposition. We use the approach introduced in [69], namely, we consider dynamics in complex time.

\subsection{Domain in the complex time plane}

Let $\lambda_{j}(\kappa), j=1, \ldots, n$ be eigenvalues of the equilibrium $X(\kappa)$. Fix a solution $\kappa=$ $\mathrm{K}(\tau), \tau=\varepsilon t \in\left[\tau_{0}, \tau_{1}\right]$ of the slow system, and consider the equilibrium of the fast system and its eigenvalues along this solution. Let $\tau_{*} \in\left(\tau_{0}, \tau_{1}\right)$ be the (slow) time moment of the stability loss in the quasi-static setting, and let $\lambda_{1}(\mathrm{~K}(\tau))$ and $\lambda_{2}(\mathrm{~K}(\tau))=\bar{\lambda}_{1}(\mathrm{~K}(\tau))$ be the eigenvalues responsible for stability loss. Thus, we assume that $\lambda_{1,2}(\mathrm{~K}(\tau))$ are in the left complex half-plane for $\tau<\tau_{*}$, they cross the imaginary axis at $\tau=\tau_{*}$ with a non-zero speed, and $\lambda_{1,2}\left(\mathrm{~K}\left(\tau_{*}\right)\right) \neq 0$. The remaining eigenvalues $\lambda_{j}(\mathrm{~K}(\tau)), j=3, \ldots, n$ are in the left half plane for all $\tau \in\left[\tau_{0}, \tau_{1}\right]$.

We assume that the right hand sides of equations (1.4) can be continued analytically in $x, \kappa$ and as smooth functions in $\varepsilon$ in a complex neighbourhood of the point $\left(X\left(\mathrm{~K}\left(\tau_{*}\right)\right), \mathrm{K}\left(\tau_{*}\right)\right)$, and this neighbourhood does not depend on $\varepsilon$. According to Theorem 3 , in this situation a stability loss delay appears.

Assuming without loss of generality that $\operatorname{Im} \lambda_{1}\left(\mathrm{~K}\left(\tau_{*}\right)\right)<0$, we introduce the function

$$
\Psi(\tau)=\int_{\tau_{*}}^{\tau} \lambda_{1}(K(\vartheta)) d \vartheta, \quad \tau \in\left[\tau_{0}, \tau_{1}\right]
$$

The function $\operatorname{Re} \Psi$ has a minimum at $\tau=\tau_{*}$ since $\operatorname{Re} \lambda_{1}(\mathrm{~K}(\tau))$ changes its sign from negative to positive at this point. For any $\tau_{-}<\tau_{*}$ close enough to $\tau_{*}$ we can define a unique moment of time $\Pi\left(\tau_{-}\right)>\tau_{*}$ such that $\operatorname{Re} \Psi\left(\Pi\left(\tau_{-}\right)\right)=\operatorname{Re} \Psi\left(\tau_{-}\right)$.

The slow solution can be analytically continued into some neighbourhood of the point $\tau_{*}$ in the plane of the complex slow time $\tau$. The analytic continuation of the function $\Psi$ is well defined in this neighbourhood. Points $\tau_{-}$and $\Pi\left(\tau_{-}\right)$are connected by an arc $\Gamma_{\tau_{-}}$of the level curve $\operatorname{Re} \Psi(\tau)=$ const in the upper half-plane. Denote $\mathrm{D}_{\tau_{-}}$the domain in the complex plane bounded by $\Gamma_{\tau_{-}}$and its complex conjugate $\bar{\Gamma}_{\tau_{-}}$(Fig. 7 ). If $\tau_{-}$is sufficiently close to $\tau_{*}$, then in the domain $\mathrm{D}_{\tau_{-}}$the following conditions are satisfied:

(1) the slow solution $(X, \mathrm{~K})$ is analytic;

(2) the right hand sides of equations (1.4) are analytic at the points of the slow solution;

(3) $\lambda_{1,2}(\mathrm{~K}) \neq 0$

(4) $\lambda_{1}(\mathrm{~K}) \neq \lambda_{2}(\mathrm{~K}), \lambda_{1,2}(\mathrm{~K}) \neq \lambda_{j}(\mathrm{~K}), j=3, \ldots, n$;

(5) $\operatorname{Re} \lambda_{j}(\mathrm{~K})<0, j=3, \ldots, n$ for real $\tau$, and the linearized near the equilibrium fast system considered along any curve $\operatorname{Re} \Psi=$ const has $n-2$ eigenvalues with negative real parts, corresponding to the eigenvalues $\lambda_{j}, j=3, \ldots, n$;

(6) the tangent lines to the curves $\operatorname{Re} \Psi=$ const are not vertical.

Let $\tau_{*}^{-}$be the lower bound of the values $\tau_{-}$for which conditions $(1)-(6)$ hold for $\mathrm{D}_{\tau_{-}}$. We denote

$$
\tau_{*}^{+}=\sup _{\tau_{*}^{-}<\tau<\tau_{*}} \Pi(\tau), \quad \mathrm{D}_{*}=\bigcup_{\tau_{*}^{-}<\tau_{-}<\tau_{*}} \mathrm{D}_{\tau_{-}} .
$$




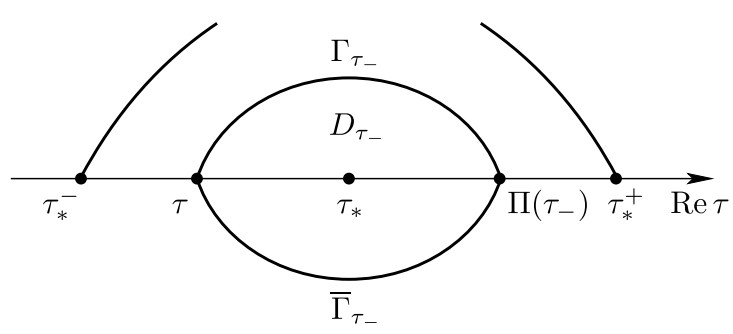

Figure 7: Domain $D_{\tau_{-}}$

\subsection{Time of stability loss delay}

Let $(x(t), \kappa(t))$ be the solution of the system (1.4) with the initial condition $\kappa_{0}=\mathrm{K}\left(\tau_{0}\right), t_{0}=$ $\tau_{0} / \varepsilon, \tau_{0}>\tau_{*}^{-}$. Denote $\beta_{0}, \gamma_{0}$ the projections of the vector $x\left(t_{0}\right)-X\left(\kappa_{0}\right)$ onto the eigenspaces of the matrix $\partial f\left(X\left(\kappa_{0}\right)\right) / \partial x$ corresponding to eigenvalues $\lambda_{1,2}$ and $\lambda_{3}, \ldots, \lambda_{n}$ respectively.

Theorem 5 [54] Suppose $\tau_{0}>\tau_{*}^{-}$and the initial point $\left(x_{0}, \kappa_{0}\right)$ lies in a $C_{1}^{-1}$-neighbourhood of the equilibrium $\left(X\left(\kappa_{0}\right), \kappa_{0}\right)$. Then for $\tau_{0}+C_{2} \varepsilon|\ln \varepsilon| \leq \varepsilon t \leq \Pi\left(\tau_{0}\right)-C_{3} \varepsilon|\ln \varepsilon|$ the phase point $(x(t), \kappa(t))$ is in the $C_{4} \varepsilon$-neighbourhood of the equilibrium $(X(\mathrm{~K}(\varepsilon t)), \mathrm{K}(\varepsilon t))$. If, additionally, $\left|\beta_{0}\right|>C_{5} \varepsilon$ and $\left|\gamma_{0}\right|<C_{6} \varepsilon$, then for $\varepsilon t=\Pi\left(\tau_{0}\right)+C_{7} \varepsilon|\ln \varepsilon|$ the phase point is outside the $C_{8}^{-1}$-neighbourhood of the equilibrium.

Remark. Condition (6) in the definition of $\tau_{*}^{-}$can be relaxed. It is sufficient to require that there exists a family of nonintersecting smooth curves which covers $\mathrm{D}_{\tau_{-}}$, and furthermore, curves from this family are symmetric with respect to the real axis and are transversal to the lines $\operatorname{Re} \Psi=$ const.

\subsection{Proof of Theorem 5}

For simplicity of the exposition, the proof is presented here for the case $n=2$. The general case is considered in [54].

Given $\tau_{-} \in\left(\tau_{*}^{-}, \tau_{0}\right)$ let $\Gamma=\Gamma_{\tau_{-}}, \mathrm{D}=\mathrm{D}_{\tau_{-}}, \xi=\left(\xi_{1}, \xi_{2}\right)=x-X(\kappa)$. We consider the complex curve

$$
L=\{\xi, \kappa: \xi=0, \kappa=\mathrm{K}(\tau), \tau \in \mathrm{D}\}
$$

\subsubsection{Preliminary transformations}

Lemma 5.1 In a $c_{1}^{-1}$-neighbourhood of $L$ there exists a real-analytic transformation of variables $\xi, \kappa \mapsto \hat{\xi}, \hat{\kappa}$ which differs by $O(\varepsilon)$ from a linear transformation of $\xi$ and from the identical transformation of $\kappa$, such that system (1.4) in the new variables takes the form (we omit the "hats" over the new variables)

$$
\begin{gathered}
\dot{\xi}=A(\kappa, \varepsilon) \xi+O\left(|\xi|^{2}\right)+O\left(\varepsilon^{3}\right), \quad \dot{\kappa}=\varepsilon F(\kappa, \varepsilon)+\varepsilon O\left(|\xi|^{2}\right)+O\left(\varepsilon^{3}\right) \\
F=g(X(\kappa), \kappa, 0)+O(\varepsilon), \quad A=\frac{1}{2}\left(\begin{array}{cc}
\Lambda_{1}+\Lambda_{2} & i\left(\Lambda_{1}-\Lambda_{2}\right) \\
i\left(\Lambda_{2}-\Lambda_{1}\right) & \Lambda_{1}+\Lambda_{2}
\end{array}\right) .
\end{gathered}
$$


The matrix $A$ has the eigenvalues $\Lambda_{i}(\kappa, \varepsilon)=\lambda_{i}(\kappa)+O(\varepsilon), i=1,2$.

Proof. In the variables $\xi, \kappa$ the equation (1.4) has the form

$$
\begin{gathered}
\dot{\xi}=\mathcal{A}(\kappa) \xi+O\left(|\xi|^{2}\right)+O(\varepsilon), \quad \dot{\kappa}=\varepsilon G(\kappa)+\varepsilon O(|\xi|)+O\left(\varepsilon^{2}\right), \\
\mathcal{A}=\partial f(X(\kappa), \kappa, 0) / \partial x, \quad G=g(X(\kappa), \kappa, 0) .
\end{gathered}
$$

Let $U$ be a small neighbourhood of the curve $L$ such that in $U$ we have $\lambda_{1,2}(\kappa) \neq 0$ and $\lambda_{1}(\kappa) \neq \lambda_{2}(\kappa)$.

The required transformation is the composition of the following ones.

A). In the equation for $\xi$ in (5.4), the free term (the term which does not vanish at $\xi=0)$ is $O(\varepsilon)$. Let us make a transformation of variables such that in the new variables the free term is $O\left(\varepsilon^{3}\right)$. For each $\kappa \in U$ the right hand side of the equation for $\xi$ in (5.4) vanishes at a unique point $\xi=\varepsilon u(\kappa, \varepsilon)$. Introduce the new variable $\hat{\xi}=\xi-\varepsilon u(\kappa, \varepsilon)$. In the equation for $\hat{\xi}$ the free term is $O\left(\varepsilon^{2}\right)$. For the obtained system, the analogous transformation leads to the free term $O\left(\varepsilon^{3}\right)$.

B). Reduce the matrix of the linearized near $\xi=0$ equation for $\xi$ to the form (5.2) with the accuracy $O\left(\varepsilon^{3}\right)$. For each $\kappa \in U$ the matrix $\mathcal{A}(\kappa)$ is similar to some matrix $\Lambda(\kappa)$ of the required form. The corresponding similarity transformation and the matrix $\Lambda$ can be chosen to be real-analytic in $\kappa$. This transformation reduces the equation for $\xi$ to such form that the matrix of the linearized near $\xi=0$ equation for $\xi$ is $\Lambda(\kappa)+O(\varepsilon)$. This matrix is similar to some matrix $\hat{\Lambda}(\kappa, \varepsilon)=\Lambda(\kappa)+O(\varepsilon)$ of the required form, and the corresponding similarity transformation can be chosen to be real-analytic and $O(\varepsilon)$-close to the identity transformation. In the new variables the matrix of the linearized equation is $\hat{\Lambda}(\kappa, \varepsilon)+O\left(\varepsilon^{2}\right)$. Making the analogous transformation of variables for this matrix we obtain the matrix of the linearized system $A(\kappa, \varepsilon)+O\left(\varepsilon^{3}\right)$, where $A(\kappa, \varepsilon)$ satisfies (5.3).

C). Eliminate, with an accuracy $O\left(\varepsilon^{3}\right)$, linear in $\xi$ terms in equation for $\kappa$. To this end make a transformation of variables $\hat{\kappa}=\kappa+\varepsilon M(\kappa, \varepsilon) \xi$, where $M$ is a matrix to be determined. The new variable $\hat{\kappa}$ satisfies the equation (we omit "hats")

$$
\dot{\kappa}=\varepsilon G(\kappa)+\varepsilon M A \xi+\varepsilon O(|\xi|)+O\left(\varepsilon^{2}\right) .
$$

Since $A$ is nonsingular, the matrix $M$ can be selected to eliminate linear in $\xi$ terms of order $\varepsilon$. In the obtained equation, an analogous transformation of variables eliminates linear in $\xi$ terms of order $\varepsilon^{2}$.

Lemma 5.2 For system (5.2), in a $c_{1}^{-1}$-neighbourhood of $L$, one can make a real-analytic transformation of variables $\hat{\xi}=\xi+O\left(|\xi|^{2}\right)$ that eliminates some quadratic in $\xi$ terms. The transformed system is such that if instead of the new variables $\xi_{1}, \xi_{2}$ ("hats" are omitted) the variables $z=\xi_{1}+i \xi_{2}, w=\xi_{1}-i \xi_{2}$ are used then right hand sides of the equations for $z$ and $w$ at $\varepsilon=0$ contain no quadratic monomials which include $z$ and $w$, respectively.

Proof. For $\Lambda_{1,2} \neq 0$, these quadratic terms are non-resonant. They can be eliminated according to the Poincaré-Dulac theorem ([4], p. 168). 


\subsubsection{Real-analyticity condition}

Let $\xi(t), \kappa(t)$ be a solution of the system obtained from (5.2) after transformation from Lemma 5.2. Consider the real initial conditions $\xi\left(t_{0}\right), \kappa\left(t_{0}\right)$.

Lemma 5.3 Suppose in the plane of the complex time $t$ the solution $\xi(t), \kappa(t)$ can be continued analytically into a neighbourhood $U$ of a real interval. Then it can be continued analytically in $\bar{U}$, and $\xi(\bar{t})=\overline{\xi(t)}, \kappa(\bar{t})=\overline{\kappa(t)}$ for $t \in U \cup \bar{U}$.

The proof is evident.

Introduce $z=\xi_{1}+i \xi_{2}, w=\xi_{1}-i \xi_{2}$ and rewrite the system in the variables $z, w, \kappa$. Lemma 5.3 implies that for real-analytic solutions $w(t)=\overline{z(\bar{t})}$. This equation will be used instead of the differential equation for $w$. We have

$$
\dot{z}=\Lambda_{1}(\kappa) z+O\left(|w|^{2}+|z|^{3}+|z|^{2}|w|+\varepsilon^{3}\right), \quad \dot{\kappa}=\varepsilon F(\kappa)+O\left(\varepsilon|\xi|^{2}+\varepsilon^{3}\right) .
$$

The argument $\varepsilon$ is omitted in the right hand sides of these equations for brevity. Instead of the system of $k+2$ ordinary differential equations we obtain a system of $k+1$ delay differential equations with an imaginary non-constant value of the delay.

\subsubsection{Initial part of motion}

Let $z(t), \kappa(t)$ be a solution of system (5.5). According to [85], p.55, if the initial point is in $C_{1}^{-1}$-neighbourhood of the equilibrium, then for some $t_{1}=t_{0}+O(|\ln \varepsilon|)$ the solution comes to an $O(\varepsilon)$-neighbourhood of the equilibrium. Take the constant $C_{5}$ such that if $\left|\beta_{0}\right|>C_{5} \varepsilon$ then $\left|z\left(t_{0}\right)\right|>\varepsilon$ (notation $\beta_{0}$ is introduced at the beginning of Section 5.2). In case if $\beta_{0}>C_{5} \varepsilon$ we can take as $t_{1}$ the first moment of time $t>t_{0}$ for which $|z(t)|=\varepsilon$.

\subsubsection{Preliminary construction for the principal part of motion}

Let $\mathrm{K}_{\varepsilon}(\tau)$ be the solution of the modified slow equation $\dot{\mathrm{K}}=\varepsilon F(\mathrm{~K})$ with the initial condition $\mathrm{K}_{\varepsilon}\left(\varepsilon t_{1}\right)=\kappa\left(t_{1}\right)$. Introduce the "phase" $\Psi_{\varepsilon}(\tau)=\int_{\tau_{\star}}^{\tau} \Lambda_{1}\left(\mathrm{~K}_{\varepsilon}(\vartheta)\right) d \vartheta$ and consider in the upper half plane of the complex variable $\tau$ arcs of level curves $\operatorname{Re} \Psi_{\varepsilon}=$ const with endpoints on the real axis. Let $\Gamma_{\varepsilon}$ be the arc with the left endpoint $\varepsilon t_{1}$. Denote $\Pi_{\varepsilon}\left(\varepsilon t_{1}\right)$ the right endpoint of this arc. Then $\Pi_{\varepsilon}\left(\varepsilon t_{1}\right)=\Pi\left(\tau_{0}\right)+O(|\varepsilon \ln \varepsilon|)$. Let $\mathrm{D}_{\varepsilon}$ be the domain in the plane of variable $\tau$ bounded by the $\operatorname{arcs} \Gamma_{\varepsilon}$ and $\bar{\Gamma}_{\varepsilon}$. Denote $S(T)$ the part (a sector) of this domain to the left of the line $\operatorname{Re} t=T$.

\subsubsection{The principal part of the motion}

We have $\left|z\left(t_{1}\right)\right|<c_{2} \varepsilon$. By Cauchy's theorem [33] there exists $T>0$ such that the solution $z(t), \kappa(t)$ can be continued analytically into a sector $S(T)$, and in this sector

$$
|z(t)|<2 c_{2} \varepsilon, \quad\left|\kappa(t)-\mathrm{K}_{\varepsilon}(\varepsilon t)\right|<\varepsilon .
$$

Let $T_{1}$ be the upper bound of the values $T$ such that the solution can be analytically continued to the sector $S(T)$ and inequalities (5.6) are satisfied. Denote $S_{1}=S\left(T_{1}\right)$. Our objective is to prove that $\varepsilon T_{1}=\Pi_{\varepsilon}\left(\varepsilon t_{1}\right)$. 
First, we estimate $\kappa(t)$ for $t \in S_{1}$. According to (5.5),(5.6) we have $\dot{\kappa}=\varepsilon F(\kappa)+O\left(\varepsilon^{3}\right)$. Thus $\left|\kappa(t)-\mathrm{K}_{\varepsilon}(\varepsilon t)\right|=O\left(\varepsilon^{2}\right)<\frac{1}{2} \varepsilon$.

Now we estimate $z(t)$ for $t \in S_{1}$. According to (5.5), (5.6) we have

$$
\dot{z}=\Lambda_{1}(\kappa) z+\alpha, \quad \alpha=O\left(\varepsilon^{2}\right) .
$$

Each point of the sector $S_{1}$ can be reached from $t_{1}$ by moving along upper boundary $\Gamma_{\varepsilon}$ of $S_{1}$ and then vertically downwards.

For the motion along $\Gamma_{\varepsilon}$ equation (5.7) takes the form

$$
\frac{d z}{d \sigma}=i \omega(\varepsilon \sigma) z+O(\alpha)
$$

where $\sigma$ is the arc length along $\Gamma_{\varepsilon}$ and $\omega$ is a real function separated from 0 by a positive constant $\tilde{c}_{3}^{-1}$. The function $\omega$ is real because $\operatorname{Re} \Psi_{\varepsilon}$ does not change along $\Gamma_{\varepsilon}$; this property plays a basic role for the delay phenomenon. Thus on $\Gamma_{\varepsilon}$ for $t_{1} \leq \operatorname{Re} t \leq T_{1}$ we have

$$
\frac{d|z|^{2}}{d \sigma}=O(|z| \alpha)=O\left(\varepsilon^{3}\right)
$$

Therefore, on $\Gamma_{\varepsilon}$ we have $|z(t)|<\frac{3}{2} c_{2} \varepsilon$ at least for $\varepsilon \operatorname{Re} t \leq \min \left\{\varepsilon T_{1}, \varepsilon t_{1}+c_{3}^{-1}\right\}$.

For the motion downwards along a vertical line Re $t=$ const equation (5.7) takes the form

$$
\frac{d z}{d s}=-i \Lambda_{1}\left(\mathrm{~K}_{\varepsilon}(\varepsilon t)\right) z+O(\alpha), \quad s=-\operatorname{Im} t
$$

According to condition 6) in Section 5.1, each vertical line in $S_{1}$ crosses the curves $\operatorname{Re} \Psi_{\varepsilon}=$ const transversally. Thus $\operatorname{Re} \Psi_{\varepsilon}$ decreases downwards along this line, and the real part of the function $-i \Lambda_{1}\left(\mathrm{~K}_{\varepsilon}(\varepsilon t)\right)$ is negative and separated from 0 by a constant. This implies that $|z(t)|$ decreases for motion downwards along any line $\operatorname{Re} t=$ const while $|z(t)|>c_{4} \varepsilon^{2}$. Therefore, $|z(t)|<\frac{3}{2} c_{2} \varepsilon$ on these lines. Hence, conditions (5.6) are satisfied with some reserve at least for $\varepsilon t_{1} \leq \varepsilon \operatorname{Re} t \leq \varepsilon t_{1}+c_{3}^{-1}$. Thus $\varepsilon T_{1}>\varepsilon t_{1}+c_{3}^{-1}$.

For $t_{1}+c_{5}|\ln \varepsilon|<\operatorname{Re} t<\varepsilon^{-1} \Pi_{\varepsilon}\left(t_{1}\right)-c_{5}|\ln \varepsilon|$ the segment of the line Re $t=$ const in $S_{1}$ is long enough for $|z(t)|$ to decay at least to $c_{6} \varepsilon^{2}$. In this case $|z(t)|=O\left(\varepsilon^{2}\right)$ for $t \in \bar{\Gamma}_{\varepsilon}$. As $w(t)=\overline{z(\bar{t})}$ we obtain that $|w(t)|=O\left(\varepsilon^{2}\right)$ for $t \in \Gamma_{\varepsilon}$. Then, according to (5.5),(5.6), for motion along $\Gamma_{\varepsilon}$ equation (5.7) takes the form (5.8) with improved estimate for the last term: $\alpha=O\left(\varepsilon^{3}\right)$ for $t_{1}+c_{5}|\ln \varepsilon|<\operatorname{Re} t<\varepsilon^{-1} \Pi_{\varepsilon}\left(t_{1}\right)-c_{5}|\ln \varepsilon|$ and $\alpha=O\left(\varepsilon^{2}\right)$ otherwise (all under assumption that $\operatorname{Re} t \leq T_{1}$ ). For motion along $\Gamma_{\varepsilon}$, equation (5.9) implies that

$$
|z(t)|=\left|z\left(t_{1}\right)\right|+O\left(\varepsilon^{2} \ln \varepsilon\right)<\frac{3}{2} c_{2} \varepsilon .
$$

Consider again the motion downwards along lines Re $t=$ const starting from points of $\Gamma_{\varepsilon}$. Equations (5.10), (5.11) imply that $|z(t)|<\frac{3}{2} c_{2} \varepsilon$ along these lines.

Thus, for $t \in S_{1}$ inequalities (5.6) are satisfied with a reserve. If $\varepsilon T_{1}<\Pi_{\varepsilon}\left(\varepsilon t_{1}\right)$ then the Cauchy theorem implies that the solution $z(t), \kappa(t)$ can be analytically continued to a sector $S(T), T>T_{1}$, in which inequalities (5.6) are still satisfied. This contradicts the definition of $T_{1}$. Thus $\varepsilon T_{1}=\Pi_{\varepsilon}\left(\varepsilon t_{1}\right)=\Pi\left(\tau_{0}\right)+O(\varepsilon|\ln \varepsilon|)$. Using inequalities (5.6) for real $t$ and returning to the original variables we obtain the first conclusion of the theorem. 


\subsubsection{The escape part of the motion}

Let $\beta_{0}>C_{5} \varepsilon$. Then, according to Sec. 5.3.3, $\left|z\left(t_{1}\right)\right|=\varepsilon$. Then, according to Sec. 5.3.5, for $T_{1}=\varepsilon^{-1} \Pi_{\varepsilon}\left(\varepsilon t_{1}\right)$ we have

$$
\left|z\left(T_{1}\right)\right|=\left|z\left(t_{1}\right)\right|+O\left(\varepsilon^{2} \ln \varepsilon\right)>\frac{1}{2} \varepsilon, \quad\left|z\left(T_{1}\right)\right|=O(\varepsilon), \quad|\kappa(t)-\mathrm{K}(\varepsilon t)|=O(\varepsilon) .
$$

Equations (5.5) imply that in the domain

$$
\frac{1}{4} \varepsilon \leq|z| \leq c_{7}^{-1}, \quad\left|\kappa-\mathrm{K}\left(\varepsilon T_{1}\right)\right| \leq c_{8}^{-1}
$$

we have

$$
c_{9}^{-1}|z|^{2}<d|z|^{2} / d t<c_{9}|z|^{2}, \quad \dot{\kappa}=O(\varepsilon) .
$$

Hence, while the phase point moves in the domain (5.12) we have

$$
|z(t)|>\frac{1}{2} \varepsilon \exp \left(c_{9}^{-1}\left(t-T_{1}\right) / 2\right), \quad \kappa(t)=\mathrm{K}\left(\varepsilon T_{1}\right)+O(\varepsilon)\left(t-T_{1}+1\right) .
$$

Let $T_{2}$ be the maximum time such that for $t \in\left[T_{1}, T_{2}\right]$ inequalities (5.12) and $|z| \leq \frac{1}{2} c_{7}^{-1}$ are satisfied. Estimates (5.13) imply that $T_{2}<T_{1}+O(\ln \varepsilon)$ and that at $t=T_{2}$ the phase point is inside the domain (5.12). Therefore $z\left(T_{2}\right)=\frac{1}{2} c_{7}^{-1}$, which implies the second conclusion of the theorem.

\section{On maximal delay (buffer point) for an equilibrium of fast system}

The phenomenon of maximal delay ( a buffer point or butée, see the end of Section 4) was discovered by Pontryagin and Shishkova on a model example in [69]. The maximal delay is controlled by closest to the real axis (in some natural sense) singularities of systems (1.4), (1.6) in the complex time plane. We assume that the functions $f, g$ in the right hand side of the system (1.4) can be analytically continued to a large enough complex neighbourhood of $\mathcal{X} \times \mathcal{K}$. So the singularities of these functions are not important here. Then typical important singularities occur when equilibrium $X(\kappa)$ has either zero eigenvalue (in this case typically the equilibrium has a second order branching as a function of the variable

$\kappa)$ or equal eigenvalues (in this case typically the eigenvalue has a second order branching as a function of the variable $\kappa$ ).

In [69] the following example is considered

$$
\dot{z}=(\kappa-i)(z-\kappa)+\gamma(z-\kappa)^{2}(\bar{z}-\kappa), \quad \dot{\kappa}=\varepsilon, \quad \gamma=\text { const. }
$$

Here $z$ is a complex variable, $z=x_{1}+i x_{2}$. This is a slow-fast system with two real fast variables and one slow variable. We can take $\kappa$ as a slow time: $\kappa=\tau$. For fixed $\tau$ the system has an equilibrium at $z=\tau$. Eigenvalues of this equilibrium are $\lambda=\tau-i$ and $\bar{\lambda}$. The equilibrium loses its stability at $\tau=0$. It is a stable focus for $\tau<0$ and an unstable focus for $\tau>0$. 


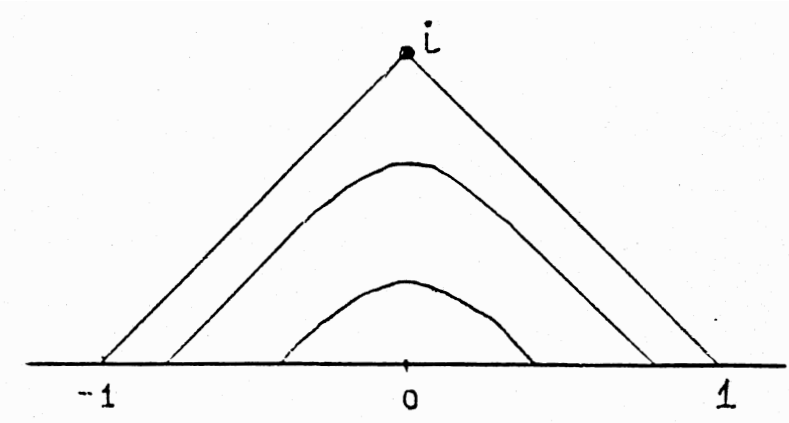

Figure 8: Level curves of the function Re $\Psi$ in Pontryagin-Shishkova example

Introduce the complex phase $\Psi$ according to (5.1):

$$
\Psi(\tau)=\int_{0}^{\tau}(\vartheta-i) d \vartheta=\frac{1}{2}(\tau-i)^{2}+\frac{1}{2} .
$$

The level curves of the function $\operatorname{Re} \Psi$ for complex $\tau$ are shown in Fig. 8. The eigenvalue $\lambda=\tau-i$ vanishes at $\tau=i$. Passing through this point level curves of the function $\operatorname{Re} \Psi$ cross the real axis at the points $\tau_{*}^{-}=-1$ and $\tau_{*}^{+}=1$. It is shown in [69] that phase points, attracted to the equilibrium of the fast system at $\tau<-1$, escape from $O(1)$-neighbourhood of this equilibrium near the moment of slow time $\tau=1$.

Equation (6.1) for $\tau=$ const looks as a quite typical example from the viewpoint of static bifurcation theory, as it is a normal form for Poincaré-Andronov-Hopf bifurcation. However, it is not a typical example from the viewpoint of dynamic bifurcation theory. The eigenvalue $\lambda=\tau-i$ of the equilibrium $z=z_{e}=\tau$ vanishes at $\tau=i$, but $z_{e}$ is an analytic function at this point. On the other hand, thanks to this, the analysis of this example provides a very transparent picture of the maximal delay phenomenon.

Below we consider calculation of the maximal delay in an example with a typical singularity (second order branching) at the point where an eigenvalue of the equilibrium vanishes. In this example the equation arises as a normal form for the motion in the complex time near the point where an eigenvalue vanishes. Analysis of this system plays a central role in the study of maximal delay in the case of such a singularity [55] ${ }^{7}$.

Consider the system

$$
\dot{z}=z^{2}+i(\kappa-i), \quad z=x_{1}+i x_{2}, \quad \dot{\kappa}=\varepsilon .
$$

This is a slow-fast system of differential equations for real fast variables $x_{1}, x_{2}$ and the slow variable $\kappa$. We can take $\kappa$ as a slow time: $\kappa=\tau$. For fixed $\tau$ the system has equilibria at $z=z_{e}(\tau)=\sqrt{-i(\tau-i)}$. Since the square root has two values, there are two equilibria. We will interested in one of them, having $\operatorname{Im} z_{e}<0$. The eigenvalues of the equilibrium $z_{e}$ are $\lambda=2 z_{e}$ and $\bar{\lambda}$. This equilibrium loses stability at $\tau=0$. It is a stable focus for $\tau<0$ and an unstable focus for $\tau>0$.

\footnotetext{
${ }^{7} \mathrm{~A}$ general theorem about maximal delay in this case is formulated in [55]. The proof is not published.
} 


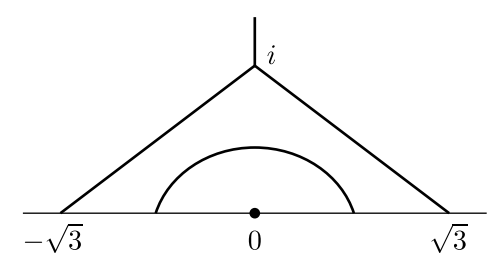

Figure 9: Level curves of function $\operatorname{Re} \Psi$ for equation (6.2)

Consider the system (6.2) for complex time $t$ and, therefore, for complex $\tau$. The point $\tau=i$ is a branching point for the equilibrium. At this point $\lambda=0$. Denote

$$
\Psi(\tau)=\int_{0}^{\tau} \lambda d \tau=\frac{4}{3} \sqrt{-i}(\tau-i)^{3 / 2}+\frac{4}{3} .
$$

Level curves Re $\Psi=$ const are shown in Fig. 9. The level curves adjacent to the critical point $\tau_{c}=i$ are Stokes lines. Angles between them at the critical point $\tau_{c}=i$ are equal to $2 \pi / 3$. The Stokes lines cross real axis at the points $\tau_{*}^{-}=-\sqrt{3}$ and $\tau_{*}^{+}=\sqrt{3}$. Consider the system (6.2) when $\tau$ changes along the line segments $[ \pm \sqrt{3}, i]$ joining points $\pm \sqrt{3}$ with point $i$. As a result of the transformation

$$
t=i \mp \vartheta e^{\mp i \pi / 6}, \quad s=\varepsilon \vartheta, \quad z=\xi e^{\mp i \pi / 3}
$$

the system (6.2) takes the same form for both segments:

$$
\frac{d \xi}{d \vartheta}=i\left(\xi^{2}+s\right), \quad \frac{d s}{d \vartheta}=\varepsilon .
$$

In (6.3) one should choose the upper sign for the segment $[\sqrt{3}, i]$ and the lower sign for the segment $[-\sqrt{3}, i]$. The variable $\vartheta$ is real, and $\vartheta<0$ for both segments. Rescaling

$$
s=\varepsilon^{2 / 3} \tilde{s}, \quad \xi=\varepsilon^{1 / 3} \tilde{\xi}
$$

transforms system (6.4) into

$$
\frac{d \tilde{\xi}}{d \tilde{s}}=i\left(\tilde{\xi}^{2}+\tilde{s}\right) .
$$

This Riccati equation is well known in the theory of relaxation oscillations (see [49] and references therein). In this theory it appears without the coefficient $i$ in front of the right hand side. In complex time this equation is considered in [16]. It does not contain a small parameter. The general solution of this equation is (see [49])

$$
\tilde{\xi}=-i \sqrt{-\tilde{s}} \frac{J_{-2 / 3}(w)-R J_{2 / 3}(w)}{R J_{-1 / 3}(w)+J_{1 / 3}(w)}, \quad w=\frac{2}{3}(-\tilde{s})^{3 / 2} .
$$

Here $J_{\nu}(\cdot)$ is the Bessel function and $R$ is a constant determined by initial conditions.

Consider any solution of system (6.2). At the segment $[-\sqrt{3}, i]$ it has the form (6.6) with some $R=R_{l}$. At the segment $[\sqrt{3}, i]$ the same solution has the form (6.6) with some 
$R=R_{r}$. The relation between $R_{l}$ and $R_{r}$ follows from transformation formulas (6.3) and asymptotic formulas for Bessel functions at the point $w=0,[34]$ :

$$
R_{r}=R_{l} e^{-2 \pi i / 3}
$$

The system (6.2) has two special solutions which have asymptotic behavior $z \sim z_{e}$, respectively, as $t \rightarrow-\infty$ and $t \rightarrow+\infty$. The first of these solutions has asymptotic behaviour $\tilde{\xi}=-\sqrt{-\tilde{s}}(1+O(1 /|w|))$ along the segment $[-\sqrt{3}, i]$. This solution is given by (6.6) with $R=R_{-}=e^{2 \pi i / 3}$ (to calculate this value of $R$ one should substitute asymptotic formulas for Bessel functions with large $|w|,[34]$, into (6.6)). The second special solution has asymptotic behaviour $\tilde{\xi}=\sqrt{-\tilde{s}}(1+O(1 /|w|))$ along the segment $[\sqrt{3}, i]$. This solution is given by (6.6) with $R=R_{+}=e^{-2 \pi i / 3}$. These two solutions are different since (6.7) is not satisfied for the values $R_{ \pm}$. Splitting of these two solutions is the reason for the existence of the maximal delay time. The first (left) special solution, whose asymptotic behaviour along the segment $[-\sqrt{3}, i]$ is $\tilde{\xi}=-\sqrt{-\tilde{s}}(1+O(1 /|w|))$, can be presented on the segment $[\sqrt{3}, i]$ by $(6.6)$ with $R=1$ (here we have used equation (6.7)). It has poles on the segment $[\sqrt{3}, i]$ because the function $J_{-1 / 3}(w)+J_{1 / 3}(w)$ has zeroes on the positive real semi-axis [34]. This solution is an analytic function of time inside the triangle with vertices $-\sqrt{3}, i, \sqrt{3}$. Inside this triangle this solution (and any other solution which is defined on the segment $[\sqrt{3}, i]$ by formula (6.6) with $R \neq e^{2 \pi i / 3}$ ) has the expansion

$$
\tilde{\xi}=\sqrt{-\tilde{s}}\left[1-2 e^{-2 i w} e^{\pi i / 6} \frac{R-e^{-2 \pi i / 3}}{R-e^{2 \pi i / 3}}+O\left(e^{-4|\operatorname{Im} w|}+\frac{1}{|w|}\right)\right] .
$$

Inside the triangle we have $\operatorname{Im} w<0$. To obtain expansion (6.8) one should substitute into (6.6) asymptotic formulas for Bessel functions with large $|w|$ and expand the result with respect to $e^{-i w}$.

Now let us consider a solution $z(\cdot)$ of system (6.2) such that $z(t)$ is $O(1)$-close to $z_{e}(\tau)$ for some (real) $\tau=\tau_{-}<-\sqrt{3}$. For some $\tau=\tau_{-}+O(\varepsilon|\ln \varepsilon|)<-\sqrt{3}$ we have $z(t)=z_{e}(\tau)+O(\varepsilon)$. For $\tau=-\sqrt{3}$, we also have $z(t)=z_{e}(\tau)+O(\varepsilon)$. Therefore, in the variables (6.3) with the lower sign, the solution $z(t)$ is determined by (6.6) with $R=e^{2 \pi i / 3}+O(\varepsilon)$. In the variables (6.3) with the upper sign, the solution $z(t)$ is determined by (6.6) with $R=1+O(\varepsilon)$.

On the real axis for $\tau=\sqrt{3}-C \varepsilon, C=$ const $>0$, the expansion (6.8) is valid, where $|w| \sim 1 / \varepsilon, \operatorname{Im} w \sim-C$. Hence, for such $\tau$ and for big enough constant $C$ the point $z(t)$ is at the distance of order 1 from the equilibrium: $c_{1}^{-1}<\left|z(t)-z_{e}(\tau)\right|<2 c_{1}^{-1}$ (here the value of the constant $c_{1}$ is determined by the choice of the constant $C$ ). This implies that departure of the phase point $z(t)$ from the equilibrium takes place in an $O(\varepsilon|\ln \varepsilon|)$-neighborhood of $\tau=\sqrt{3}$.

Remark. For the phase points, which are attracted to the stable equilibrium in an $O(\varepsilon|\ln \varepsilon|)$-neighborhood of $\tau=-\sqrt{3}$, the moments of departure are distributed in the interval $(\sqrt{3}+O(\varepsilon|\ln \varepsilon|), \infty)$. This follows from the consideration of the motion back in time for the phase points which depart from the unstable equilibrium at $\tau>\sqrt{3}$. 


\section{On stability loss delay for a periodic trajectory}

Consider again system (1.4) under assumptions of Theorem 3. This theorem guarantees existence of the stability loss delay for the state $L_{\kappa}$ but does not give asymptotic formulas or realistic estimates for the delay time. For the case when $L_{\kappa}$ is an equilibrium, such formulas/estimates are provided by Theorem 5 on the basis of analytic continuation of solutions in the plane of complex time at the distance $\sim 1 / \varepsilon$ from the real axis. Such an approach does not work in the case when $L_{\kappa}$ is a periodic trajectory as then solutions in general cannot be analytically continued into so large domain of $\tau$.

Nevertheless, properties of the system in complex time play a principal role in the stability loss delay for a periodic trajectory. The family of periodic trajectories $L_{\kappa}$ for $\kappa=K(\tau), \tau_{0} \leq \tau \leq \tau_{1}$ forms a manifold

$$
M=\left\{(x, \kappa): x \in L_{K(\tau)}, \kappa=K(\tau), \tau_{0} \leq \tau \leq \tau_{1}\right\}
$$

Theorem 3 implies that in an $O(\varepsilon)$-neighbourhood of the piece of this manifold with $\tau \in\left(\tau_{*}-c^{-1}, \tau_{*}+c^{-1}\right), c=$ const $>0$, there exists a piece of an invariant manifold $\tilde{M}$ of the exact system (1.4). One can try to find this invariant manifold and to extend it to a larger interval $\left(\tau_{-}, \tau_{+}\right)$. Estimates of stability loss delay time can be obtained using linearization of the exact system near $\tilde{M}$. The interval of values $\tau$ on which $\tilde{M}$ stays in an $O(\varepsilon)$-neighbourhood of $M$ cannot be extended arbitrarily to both sides. The maximal size of this interval is determined by properties of the system for complex values of $\tau$. Estimates of delay time for a periodic trajectory were obtained by using such an approach in [75, 76] for the case of periodic trajectories of a time-periodic system with two other fast variables and in [56] for periodic trajectories with an arbitrary number of fast variables. In this section we expose results of [56].

\subsection{Notation, basic assumptions, and construction of a domain in the plane of complex slow time}

Consider the system (1.4) in the case when the slow variable $\kappa$ is one-dimensional and $g=1$. We can take $\kappa$ as a slow time: $\kappa=\tau$. The system takes the form

$$
\dot{x}=f(x, \tau, \varepsilon), \quad \dot{\tau}=\varepsilon .
$$

The fast system is

$$
\dot{x}=f(x, \tau, 0), \quad \tau=\text { const } .
$$

We assume that the periodic trajectory $L_{\tau}$ of the fast system exists and continuously depends on $\tau$ for any fixed $\tau \in\left[\tau_{0}, \tau_{1}\right]$. Let

$$
T(\tau), \quad \omega(\tau)=2 \pi / T(\tau), \quad \rho_{1}(\tau), \ldots, \rho_{n-1}(\tau)
$$

be the period, frequency and multipliers of $L_{\tau}$, respectively. Later we consider $L_{\tau}$ as a set in the space of the variables $x, \tau$ as well as in the space of the variables $x$. 
As in Section 4 we assume that for $\tau<\tau_{*} \in\left(\tau_{0}, \tau_{1}\right)$ the periodic trajectory $L_{\tau}$ is linearly asymptotically stable (all the multipliers are inside the unit circle), and that it loses stability at $\tau=\tau_{*}$, but remains nondegenerate. Then typically either a pair of complex conjugate multipliers $\rho_{1}$ and $\rho_{2}=\bar{\rho}_{1}$ leaves the unit circle through different points, or one real multiplier $\rho_{1}$ leaves the unit circle through the point -1 ; the other multipliers remain inside the unit circle. In what follows we consider the first of these cases (two different complex conjugate multipliers on the unit circle at $\tau=\tau_{*}$ ). The second case can be considered in an analogous way (see details in [56]). We assume that the multipliers $\rho_{1,2}$ cross the unit circle with a non-zero velocity.

We assume that the periodic trajectory $L_{\tau}$ can be continued analytically in a complex neighbourhood $U \subset \mathbb{C}$ of the point $\tau_{*}$, and that the right hand side of the fast system (7.3) is analytic in $x, \tau$ in a complex neighbourhood of $L_{\tau}$ for $\tau \in U$. We also assume that the following assumptions hold.

$C 1$. In the domain $U$ all the multipliers $\rho_{l}(\tau)$ are analytic in $\tau$ and distinct from one. The frequency $\omega$ is analytic and does not vanish in $U$.

C2. In the domain $U$ the multipliers $\rho_{l}(\tau)$ are pairwise distinct.

Without loss of generality we assume that

$$
\operatorname{Im} \rho_{1}\left(\tau_{*}\right)=-\operatorname{Im} \rho_{2}\left(\tau_{*}\right)<0 .
$$

Introduce eigenvalues. The eigenvalues $\lambda_{l}^{0}(\tau), l=1, \ldots, n-1$, are continuous functions of $\tau$ such that

$$
\rho_{l}(\tau)=\exp \left(\lambda_{l}^{0}(\tau) T(\tau)\right), \quad \operatorname{Im} \lambda_{l}^{0}\left(\tau_{*}\right) T\left(\tau_{*}\right) \in(-\pi, \pi]
$$

All other eigenvalues are defined as

$$
\lambda_{l}^{r}(\tau)=\lambda_{l}^{0}(\tau)-i r \omega(\tau), \quad r \in \mathbb{Z} \backslash\{0\}
$$

We have:

$$
\begin{aligned}
\lambda_{1}^{r}(\tau) & =\bar{\lambda}_{2}^{-r}(\bar{\tau}), \quad \tau \in U, r \in \mathbb{Z}, \\
\operatorname{Im} \lambda_{1}^{0}\left(\tau_{*}\right) T\left(\tau_{*}\right) & \in(-\pi, 0), \quad \operatorname{Re} \lambda_{1}^{r}\left(\tau_{*}\right)=\operatorname{Re} \lambda_{2}^{r}\left(\tau_{*}\right)=0 .
\end{aligned}
$$

For the sake of being definite we assume that $\left|\lambda_{1}^{0}\left(\tau_{*}\right)\right|<\left|\lambda_{1}^{-1}\left(\tau_{*}\right)\right|$; if the opposite inequality is satisfied then one should replace $\lambda_{1}^{0}$ with $\lambda_{1}^{-1}$ in the reasoning below. The eigenvalues $\lambda_{1}^{0}\left(\tau_{*}\right), \lambda_{1}^{-1}\left(\tau_{*}\right)$ are shown in Fig. 10(a).

Introduce the functions

$$
\psi_{l}^{r}(\tau)=\int_{\tau_{*}}^{\tau} \lambda_{l}^{r}(s) d s, \quad \phi_{l}^{r}(\tau)=\operatorname{Re} \psi_{l}^{r}(\tau), \quad \phi^{ \pm}(\tau)=\mp \operatorname{Re} \int_{\tau_{*}}^{\tau} i \omega(s) d s, \quad \tau \in U .
$$

We will need the following assumption.

C3. The equation $\phi^{ \pm}(\tau)=0, \tau \in U$ has only real solutions.

Let $\gamma_{l}^{r}(\sigma)$ be level lines of the functions $\phi_{l}^{r}: \gamma_{l}^{r}(\sigma)=\left\{\tau \in \mathbb{C}: \phi_{l}^{k}(\tau)=\sigma\right\}$. By $(7.7)$ the functions $\phi_{1}^{r}, \phi_{2}^{r}$ restricted to the real axis have a critical point at $\tau=\tau_{*}$. Hence for 

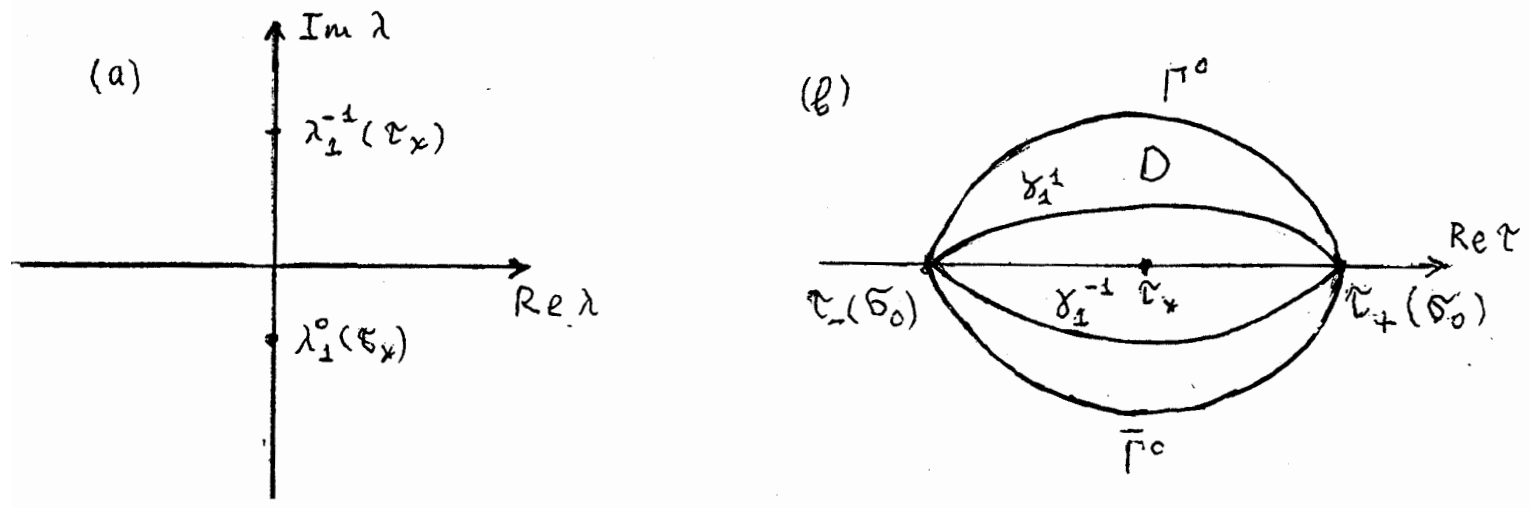

Figure 10: (a) Eigenvalues. (b) Domain $D$ and curves $\Gamma^{0}\left(\sigma_{0}\right), \bar{\Gamma}^{0}\left(\sigma_{0}\right), \gamma_{1}^{r}\left(\sigma_{0}\right)$.

each of the curves $\gamma_{l}^{r}(0), l=1,2$ the tangent line at $\tau=\tau_{*}$ is horizontal. For small enough $\sigma>0$ the curves $\gamma_{l}^{r}(\sigma), l=1,2$ intersect at the real points $\tau_{ \pm}(\sigma)$, where

$$
\tau_{-}(\sigma)<\tau_{*}<\tau_{+}(\sigma), \quad \int_{\tau_{*}}^{\tau_{ \pm}(\sigma)} \operatorname{Re} \lambda_{l}^{r}(s) d s=\sigma, \quad s \in \mathbb{R} .
$$

Let $\Gamma^{r}(\sigma)$ denote the arc of the curve $\gamma_{1}^{r}(\sigma)$ with endpoints on the real axis. One can define $\sigma_{0}>0$ such that the family of curves $\Gamma^{r}(\sigma), \sigma \in\left[0, \sigma_{0}\right]$ is well defined and has the following properties: 1) endpoints of $\Gamma^{r}(\sigma)$ are $\tau_{ \pm}(\sigma)$, 2) $\Gamma^{r}(0)$ degenerate to the point $\tau_{*}$, 3) $\Gamma^{r}(\sigma) \in U$.

Let $D$ be the closed domain in the complex plane bounded by $\Gamma^{0}\left(\sigma_{0}\right)$ and the curve $\bar{\Gamma}^{0}\left(\sigma_{0}\right)$, symmetric to it with respect to the real axis. The domain $D$ and curves $\Gamma^{0}\left(\sigma_{0}\right), \bar{\Gamma}^{0}\left(\sigma_{0}\right)$, $\gamma_{1}^{r}\left(\sigma_{0}\right)$ are shown in Fig. 10(b). We assume that the domain $D$ meets the following series of assumptions given in [56], Section $3 .^{8}$

A1. $\gamma_{1}^{-1}\left(\sigma_{0}\right) \subseteq D$

A2. $\left|\rho_{l}(\tau)\right|<1$ for $l=3, \ldots, n-1$ and $\tau \in D \cap \mathbb{R}$.

A3. Each of the functions $\phi_{l}^{r}(\tau), \phi^{ \pm}(\tau)$ (except $\phi_{0}^{1}(\tau), \phi_{0}^{2}(\tau)$ ) restricted to the boundary $\partial D$ of the domain $D$ has a unique maximum, and maxima of the functions $\left.\phi^{ \pm}(\tau)\right|_{\partial D}$ are nondegenerate.

These assumptions are certainly satisfied if $\sigma_{0}$ is small enough.

\subsection{Estimates of stability loss delay}

Define the functions

$$
\Pi:\left[\tau_{-}\left(\sigma_{0}\right), \tau_{*}\right] \rightarrow\left[\tau_{*}, \tau_{+}\left(\sigma_{0}\right)\right], \quad \hat{\Pi}:\left[\tau_{-}\left(\sigma_{0}\right), \tau_{*}\right] \rightarrow\left[\tau_{*}, \tau_{+}\left(\sigma_{0}\right)\right]
$$

\footnotetext{
${ }^{8}$ The assumption similar to assumption (6) in page 21 should be added to assumptions in [56]: the tangent lines to the curves $\phi_{1}^{0}=$ const are not vertical.
} 
by the following equations

$$
\int_{\tau_{*}}^{\tau} \operatorname{Re} \lambda_{1}^{0}(s) d s=\int_{\tau_{*}}^{\Pi(\tau)} \operatorname{Re} \lambda_{1}^{0}(s) d s, \quad \int_{\tau_{*}}^{\tau} \mu(s) d s=\int_{\tau_{*}}^{\hat{\Pi}(\tau)} \operatorname{Re} \lambda_{1}^{0}(s) d s,
$$

where $\mu(\tau)=\max _{1 \leq j \leq n-1} \operatorname{Re} \lambda_{j}^{0}(\tau)$. The functions $\Pi(\tau)$ and $\hat{\Pi}(\tau)$ evidently coincide for $\tau$ close to $\tau_{*}$. Consider a solution $x(t)$ of the system (7.2) with initial conditions at $t=t_{0}=\tau_{0} / \varepsilon, \tau_{0} \in\left[\tau_{-}\left(\sigma_{0}\right), \tau_{*}\right)$.

Theorem 6 [56] Suppose that the point $x\left(t_{0}\right)$ is inside a $C_{1}^{-1}$-neighbourhood of the periodic trajectory $L_{\tau_{0}}$. Then for $\tau_{0}+C_{2} \varepsilon|\ln \varepsilon| \leq \varepsilon t \leq \hat{\Pi}\left(\tau_{0}\right)-C_{3} \varepsilon|\ln \varepsilon|$ the solution $x(t)$ is inside a $C_{4} \varepsilon$-neighbourhood of the periodic trajectory $L_{\varepsilon t}$.

Thus, the loss of stability delays at least up to the moment $\tau \approx \hat{\Pi}\left(\tau_{0}\right)$

To calculate an asymptotics of the escape moment we need additional assumptions and notation. Suppose the following condition hold.

$$
\text { A4. } \operatorname{Re} \lambda_{j}^{0}(\tau)<\operatorname{Re} \lambda_{1}^{0}(\tau) \text { for all } j=3, \ldots, n-1 \text { and } \tau \in\left[\tau_{-}\left(\sigma_{0}\right), \tau_{*}\right] .
$$

Obviously, this assumption implies the identity $\hat{\Pi}(\tau) \equiv \Pi(\tau)$.

If the point $x\left(t_{0}\right)$ is close to the trajectory $L_{\tau_{0}}$ then there exists a unique point $x_{00} \in L_{\tau_{0}}$ closest to $x\left(t_{0}\right)$. Consider a hyperplane $\Theta_{0} \subset \mathbb{R}^{n}$ containing the point $x_{00}$ and transversal to $L_{\tau_{0}}$ at $x_{00}$. In a sufficiently small neighbourhood of the point $x_{00}$ on the hyperplane $\Theta_{0}$ the first return map of the system (7.3) with $\tau=\tau_{0}$ is well defined. The multipliers $\rho_{1}\left(\tau_{0}\right), \ldots, \rho_{n-1}\left(\tau_{0}\right)$ are eigenvalues of the linearized at $x_{00}$ first return map. Let $\Theta^{\prime}$ and $\Theta^{\prime \prime}$ be eigenspaces of the linearized first return map, which correspond to the multipliers $\rho_{1}, \rho_{2}$ and $\rho_{3}, \ldots, \rho_{n-1}$ respectively. We denote by $\beta^{\prime}$ and $\beta^{\prime \prime}$ projections of the vector $x\left(t_{0}\right)-x_{00}$ to $\Theta^{\prime}$ and $\Theta^{\prime \prime}$. If $n=3$ we put $\beta^{\prime \prime}=0$.

Theorem 7 [56] Suppose that the point $x\left(t_{0}\right)$ is inside a $C_{5}^{-1}$-neighbourhood of the periodic trajectory $L_{\tau_{0}}$. Let $\left|\beta^{\prime}\right|>C_{6} \varepsilon$ and $\left|\beta^{\prime \prime}\right|<C_{7} \varepsilon$. Then

(1) for $\tau_{0}+C_{2} \varepsilon|\ln \varepsilon| \leq \varepsilon t \leq \Pi\left(\tau_{0}\right)-C_{3} \varepsilon|\ln \varepsilon|$ the solution $x(t)$ is inside $C_{4} \varepsilon-$ neighbourhood of the periodic trajectory $L_{\varepsilon t}$.

(2) the point $x\left(t_{d}\right)$ is outside a $C_{8}^{-1}$-neighbourhood of the periodic trajectory $L_{\varepsilon t_{d}}$ for some $t_{d}$ where $\left|\varepsilon t_{d}-\Pi\left(\tau_{0}\right)\right|<C_{9} \varepsilon|\ln \varepsilon|$.

Assertion (1) of the Theorem 7 is just a repetition of Theorem 6 . According to assertion (2), a solution attracted to the family $L_{\tau}$ at $\tau \approx \tau_{0}$ escapes from $L_{\tau}$ at $\tau \approx \Pi\left(\tau_{0}\right)$.

Introducing additional assumptions one can get lower estimates for the delay time, which are better than the ones in Theorem 7 [56].

Proofs of Theorems 6 and 7 are based on construction of an invariant manifold $\tilde{M}$ of system (7.2) close to the invariant manifold $M$ (7.1) of system (7.3) for $\tau \in D$, and on study of dynamics near this manifold. These two main steps are described in the next two sections. Domain $D$ in the complex plane is introduced in Section 7.1. 


\subsection{Invariant manifold and boundary value problem over the com- plex domain}

Since for any $\tau \in U$ all the multipliers $\rho_{l}(\tau)$ are pairwise distinct and distinct from one (see assumptions C1,C2 in Section 7.1), in the vicinity of the trajectories $L_{\tau}, \tau \in U$ there exists an analytic change of the variables $\left(x_{1}, \ldots, x_{n}\right) \mapsto\left(\hat{\xi}_{1}, \ldots, \hat{\xi}_{n-1}, \hat{\varphi}\right)$ reducing the system (7.2) to the form

$$
\begin{gathered}
d \hat{\xi} / d t=\Lambda(\tau) \hat{\xi}+\Phi(\hat{\xi}, \hat{\varphi}, \tau), \quad d \hat{\varphi} / d t=\omega(\tau)+\Phi^{\prime}(\hat{\xi}, \hat{\varphi}, \tau), \\
\Lambda=\operatorname{diag}\left(\lambda_{1}^{0}, \ldots, \lambda_{n-1}^{0}\right), \quad \Phi=\left(\Phi_{1}, \ldots, \Phi_{n-1}\right)^{T}=O\left(|\hat{\xi}|^{2}\right), \quad \Phi^{\prime}=O(|\hat{\xi}|) .
\end{gathered}
$$

Here $\hat{\xi}=\left(\hat{\xi}_{1}, \ldots, \hat{\xi}_{n-1}\right)^{T}$. All the functions $\Phi^{\prime}, \Phi_{1}, \ldots, \Phi_{n-1}$ are $2 \pi$-periodic in $\hat{\varphi}$. The variables $\left(\hat{\xi}_{1}, \ldots, \hat{\xi}_{n-1}, \hat{\varphi}\right)$ are usually called the Lyapunov-Floquet coordinates. The surface $\{\hat{\xi}, \hat{\varphi}, \tau: \hat{\xi}=0, \tau \in U\}$ is invariant for the system (7.10). It is the invariant manifold $M$ (7.1) of system (7.3) presented in the Lyapunov-Floquet coordinates and considered for $\tau \in U$.

The system $(7.2)$ in the variables $\hat{\xi}, \hat{\varphi}, \tau$ has the form

$$
\begin{aligned}
d \hat{\xi} / d t & =[\Lambda(\tau)+\varepsilon \hat{B}(\hat{\varphi}, \tau, \varepsilon)] \hat{\xi}+\varepsilon \hat{h}(\hat{\varphi}, \tau, \varepsilon)+\hat{F}(\hat{\xi}, \hat{\varphi}, \tau, \varepsilon), \\
d \hat{\varphi} / d t & =\omega(\tau)+\varepsilon \hat{a}(\hat{\varphi}, \tau, \varepsilon)+\hat{F}^{\prime}(\hat{\xi}, \hat{\varphi}, \tau, \varepsilon) \\
d \tau / d t & =\varepsilon
\end{aligned}
$$

The functions $\hat{B}=\left(\hat{B}_{l k}\right), \hat{h}=\left(\hat{h}_{1}, \ldots, \hat{h}_{n-1}\right)^{T}, \hat{F}=\left(\hat{F}_{1}, \ldots, \hat{F}_{n-1}\right)^{T}, \hat{a}, \hat{F}^{\prime}$ are $2 \pi$-periodic in $\hat{\varphi}$, and the following conditions hold:

$$
\hat{F}=O\left(|\hat{\xi}|^{2}\right), \quad \hat{F}^{\prime}=O(|\hat{\xi}|) .
$$

Our goal is to construct an invariant manifold of the system (7.11) near the surface $\{\hat{\xi}=0\}$. The procedure of successive approximations in $\varepsilon$ does not allow to do this as the sequence of these approximations diverges in general (see discussion in Section 4.1). However, this procedure is useful as it allows to simplify the system for further analysis. Two steps of this procedure provide an analytic change of variables $\xi=\hat{\xi}+O(\varepsilon), \varphi=$ $\hat{\varphi}+O(\varepsilon)$, which is $2 \pi$-periodic in $\varphi$ and reduces the system (7.11) to the following form:

$$
\begin{aligned}
\dot{\xi} & =\left[\tilde{\Lambda}(\tau, \varepsilon)+\varepsilon^{2} B_{0}(\varphi, \tau, \varepsilon)\right] \xi+\varepsilon^{3} h_{0}(\varphi, \tau, \varepsilon)+F_{0}(\xi, \varphi, \tau, \varepsilon), \\
\dot{\varphi} & =\tilde{\omega}(\tau, \varepsilon)+\varepsilon^{3} a_{0}(\varphi, \tau, \varepsilon)+F_{0}^{\prime}(\xi, \varphi, \tau, \varepsilon), \\
\dot{\tau} & =\varepsilon
\end{aligned}
$$

where at any $\tau \in D$

$$
\begin{gathered}
\tilde{\Lambda}(\tau, \varepsilon)=\operatorname{diag}\left(\tilde{\lambda}_{1}(\tau, \varepsilon) \ldots, \tilde{\lambda}_{n-1}(\tau, \varepsilon)\right), \quad \tilde{\lambda}_{l}(\tau, \varepsilon)=\lambda_{l}^{0}(\tau)+O(\varepsilon), \\
\tilde{\omega}(\tau, \varepsilon)=\omega(\tau)+O(\varepsilon), \quad F_{0}=O\left(|\xi|^{2}\right), \quad F_{0}^{\prime}=O(|\xi|) .
\end{gathered}
$$

The surface $\{\xi=0\}$ is not invariant for system (7.12) if the term $h_{0}(\varphi, \tau, \varepsilon)$ is not identically zero. So the next goal is to eliminate this term by a transformation of variables. This transformation is described by the following statement. 
Lemma 7.1 (Basic lemma [56]) There exists a change of the variables

$$
\xi=\eta+\vartheta(\psi, \tau), \quad \varphi=\psi+\varkappa(\psi, \tau), \quad \vartheta=\left(\vartheta_{1}, \ldots, \vartheta_{n-1}\right)^{T}
$$

such that the functions

$$
\vartheta(\psi, \tau) / \varepsilon^{2}, \quad \varkappa(\psi, \tau) / \varepsilon \quad \text { for } \tau \in D,|\operatorname{Im} \psi|<\beta_{0} / 2
$$

are bounded and the system (7.12) in the variables $\eta, \psi, \tau$ has the form

$$
\begin{aligned}
\dot{\eta} & =\left[\tilde{\Lambda}(\tau, \varepsilon)+\varepsilon^{2} B(\psi, \tau, \varepsilon)\right] \eta+F(\eta, \psi, \tau, \varepsilon) \\
\dot{\psi} & =\tilde{\omega}(\tau, \varepsilon)+F^{\prime}(\eta, \psi, \tau, \varepsilon) \\
\dot{\tau} & =\varepsilon
\end{aligned}
$$

where the functions $B, \tilde{\omega}, F, F^{\prime}$ are analytic and bounded for

$$
|\eta| \leq \alpha_{0} / 2, \quad \tau \in D, \quad|\operatorname{Im} \psi| \leq \beta_{0} / 2,
$$

$F=O\left(|\eta|^{2}\right), F^{\prime}=O(|\eta|)$, and the constants $\alpha_{0}, \beta_{0}$ are positive.

We call a function bounded if its absolute value is bounded by a constant not depending on $\varepsilon$.

The transformation of variables (7.15) of Lemma 7.1 is constructed as a limit of a sequence of transformations of variables. At each step of the process one gets a system of equations of the form similar to (7.12). One should consider a corresponding linearized system, and find its invariant manifold. Then one should introduce deviations from this manifold as new variables. One gets for the new variables again the system of the form similar to (7.12) but with smaller term analogous to $h_{0}$. This is a realisation of the Newton method for the considered problem. Convergence of the process is ensured by quadratic decay of reminders in the Newton method. Estimates in this process are similar to those in Kolmogorov-Arnold-Moser theory [3].

The linearized system on the step number $m$ of this iteration process has the following form:

$$
\begin{aligned}
\dot{\xi}^{(m)} & =\left[\tilde{\Lambda}(\tau)+\varepsilon^{2} B_{m}\left(\varphi^{(m)}, \tau\right)\right] \xi^{(m)}+\varepsilon^{3} h_{m}\left(\varphi^{(m)}, \tau\right), \\
\dot{\varphi}^{(m)} & =\tilde{\omega}(\tau)+\varepsilon^{3} a_{m}\left(\varphi^{(m)}, \tau\right)+\left(F_{m}^{\prime}\right)_{\xi}\left(0, \varphi^{(m)}, \tau\right) \xi^{(m)}, \\
\dot{\tau} & =\varepsilon .
\end{aligned}
$$

For brevity we do not write $\varepsilon$ among the arguments of functions. Subscript $\xi$ means derivative with respect to $\xi^{(m)}$. We are looking for an invariant manifold of system (7.18) in the parametric form: $\xi^{(m)}=\vartheta(\varphi, \tau), \varphi^{(m)}=\varphi+\varkappa(\varphi, \tau)$, where the functions $\vartheta, \varkappa$ satisfy equations

$$
\begin{aligned}
\varepsilon \vartheta_{\tau}(\varphi, \tau)+\tilde{\omega}(\tau) \vartheta_{\varphi}(\varphi, \tau)-\tilde{\Lambda}(\tau) \vartheta(\varphi, \tau) & =\varepsilon^{2} B_{m}(\varphi, \tau) \vartheta(\phi, \tau)+\varepsilon^{3} h_{m}(\varphi, \tau), \\
\varepsilon \varkappa_{\tau}(\varphi, \tau)+\tilde{\omega}(\tau) \varkappa_{\varphi}(\phi, \tau) & \left.=\varepsilon^{3} a_{m}(\varphi, \tau)+\left(F_{m}^{\prime}\right)_{\xi}(0, \varphi, \tau)\right) \vartheta(\varphi, \tau) .
\end{aligned}
$$


Here the subscripts $\tau, \varphi, \xi$ denote partial derivatives. Then the variables $\xi^{(m+1)}, \varphi^{(m+1)}$ for the next step of the iteration process are given by formulas $\xi^{(m)}=\xi^{(m+1)}+\vartheta\left(\varphi^{(m+1)}, \tau\right)$, $\varphi^{(m)}=\varphi^{(m+1)}+\varkappa\left(\varphi^{(m+1)}, \tau\right)$.

Finding a bounded solution of equations (7.19) is the central element in the construction of the invariant manifold. Expand equations (7.19) in the Fourier series in $\varphi$. Denote $\vartheta^{k}(\tau), \varkappa^{k}(\tau)$ the Fourier coefficients of functions $\vartheta(\varphi, \tau), \varkappa(\varphi, \tau)$. They should satisfy the system

$$
\begin{gathered}
\varepsilon \vartheta_{\tau}^{k}+(i k \omega(\tau) I-\tilde{\Lambda}) \vartheta^{k}=\sum_{j \in \mathbb{Z}} \varepsilon^{2} B_{m}^{k-j} \vartheta^{j}+\varepsilon^{3} h_{m}^{k} \\
\varepsilon \varkappa_{\tau}^{k}+i k \tilde{\omega} \varkappa^{k}=\varepsilon^{3} a_{m}^{k}+\sum_{j \in \mathbb{Z}}\left(F_{m}^{\prime}\right)_{\xi}^{k-j}(0, \tau) \vartheta^{j} .
\end{gathered}
$$

where $B_{m}^{k}, h_{m}^{k}, a_{m}^{k}$ and $\left(F_{m}^{\prime}\right)_{\xi}^{k}$ are Fourier coefficients of functions $B_{m}, h_{m}, a_{m}$ and $\left(F_{m}^{\prime}\right)_{\xi}$ respectively; $\vartheta^{k}=\left(\vartheta_{1}^{k}, \ldots, \vartheta_{n-1}^{k}\right)^{T}, I=\operatorname{diag}(1, \ldots, 1)$.

Consider in $D$ the functions

$$
\chi^{k}(\tau)=-\operatorname{Re} \int_{\tau_{*}}^{\tau} i k \tilde{\omega}(s) d s,(k \neq 0), \quad \chi_{l}^{k}(\tau)=\operatorname{Re} \int_{\tau_{*}}^{\tau}\left(\tilde{\lambda}_{l}^{0}(s)-i k \tilde{\omega}(s)\right) d s .
$$

Points of maxima of these functions in $D$ are on $\partial D$, because $\chi^{k}$ and $\chi_{l}^{k}$ are harmonic functions. For functions $\chi^{k}, \chi_{l}^{k}$, except of $\chi_{1}^{0}, \chi_{2}^{0}$ let $\tau^{k}$ and $\tau_{l}^{k}$ be points of global maxima in $D$. For functions $\chi_{1}^{0}, \chi_{2}^{0}$, denote $\tau_{1}^{0}$ and $\tau_{2}^{0}$ any points on $\partial D$ with $\operatorname{Im} \tau>0$ and $\operatorname{Im} \tau<0$, respectively. Consider the following boundary value problem: find the solution of system (7.20) such that $\vartheta_{l}^{k}\left(\tau_{l}^{k}\right)=0, \varkappa^{k}\left(\tau^{k}\right)=0$. This boundary value problem is equivalent to the following system of integral equations

$$
\begin{aligned}
\vartheta_{l}^{k}(\tau)= & \frac{1}{\varepsilon} \int_{\tau_{l}^{k}}^{\tau} e^{\frac{1}{\varepsilon} \int_{\nu}^{\tau}\left(\tilde{\lambda}_{l}(s)-i k \tilde{\omega}(s)\right) d s}\left[\sum_{j \in \mathbb{Z}} \varepsilon^{2}\left(B_{m}^{k-j} \vartheta^{j}\right)_{l}(\nu)+\varepsilon^{3}\left(h_{m}^{k}\right)_{l}(\nu)\right] d \nu, \\
\varkappa^{k}(\tau)= & \frac{1}{\varepsilon} \int_{\tau^{k}}^{\tau} e^{-\frac{1}{\varepsilon} \int_{\nu}^{\tau} i k \tilde{\omega}(s) d s}\left[\varepsilon^{3} a_{m}^{k}(\nu)+\sum_{j \in \mathbb{Z}}\left(F_{m}^{\prime}\right)_{\xi}^{k-j}(0, \nu) \vartheta^{j}(\nu)\right] d \nu, \\
& \left(B_{m}^{k-j} \vartheta^{j}\right)_{l}=\sum_{r=1}^{n} B_{m, l, r}^{k-j} \vartheta_{r}^{j}, \quad\left(F_{m}^{\prime}\right)_{\xi}^{k-j} \vartheta^{j}=\sum_{r=1}^{n}\left(F_{m}^{\prime}\right)_{\xi_{r}}^{k-j} \vartheta_{r}^{j}
\end{aligned}
$$

The integrals in the right-hand sides of these equations can be taken along any paths with the indicated endpoints lying in the domain $D$. Since the integrands are holomorphic functions and $D$ is simple-connected, the values of the integrals do not depend on the choice of paths. Conditions, imposed on system (7.2) and the domain $D$, allow to choose such paths that for each of equations (7.22) the exponential term is bounded. The right hand side of (7.22) can be considered as an operator acting on functions $\vartheta$, $\varkappa$. This operator is contracting in an appropriate Banach space. A unique fixed point of this operator is the solution of equations (7.22).

Solution of system (7.22) provides Fourier coefficients of functions $\theta(\varphi, \tau), \varkappa(\varphi, \tau)$. Thus, one gets a solution of equations (7.19). This concludes one step of the Newton 
method. In the limit of these iterations one gets a transformation that reduces the original system (7.2) to the form (7.17). This completes the proof of Lemma 7.1. In the form (7.17), the existence of stability loss delay is evident: during the motion in the time interval $\tau_{0} \leq \varepsilon t \leq \tau_{*}$ the phase point is attracted $O\left(e^{- \text {const } / \varepsilon}\right)$-close to the invariant manifold $\{\eta=0\}$. It needs time of order $1 / \varepsilon$ after $\tau_{*}$ to leave $O(\varepsilon)$-neighbourhood of this manifold, and, thus, $O(\varepsilon)$-neighbourhood of the approximately invariant manifold $M$ (7.1). These estimates imply Theorem 6 .

\subsection{Separation of variables in the linearized system over the com- plex domain}

Calculation of asymptotics of the delay time in Theorem 7 is based on the study of dynamics near the invariant manifold $\{\eta=0\}$ of system (7.17). For this study it is convenient to separate dynamics governed by the multipliers $\rho_{1}, \rho_{2}$ from the dynamics governed by the multipliers $\rho_{3}, \ldots, \rho_{n-1}$. The lemma below describes such a separation.

Let $\mathfrak{P}$ and $\mathfrak{N}$ be the following vector subspaces of $L(n-1, \mathbb{C})$ :

$\mathfrak{P}=\left\{P=\left(P_{j k}\right) \in L(n-1, \mathbb{C}): P_{j k}=P_{k j}=0\right.$ for $\left.j=1,2, k=3, \ldots, n-1\right\}$,

$\mathfrak{N}=\left\{N=\left(N_{j k}\right) \in L(n-1, \mathbb{C}): N_{j k}=N_{k j}=0\right.$ for $j, k=1,2$, or $\left.j, k=3, \ldots, n-1\right\}$.

Obviously, $L(n-1, \mathbb{C})=\mathfrak{P} \bigoplus \mathfrak{N}$.

Lemma 7.2 (Separation Lemma [56]) Suppose that condition A4 in Section 7.2 holds. Then there exists a linear change of the variables

$$
\eta=(I+\varepsilon N(\psi, \tau, \varepsilon)) \zeta, \quad I=\operatorname{diag}(1, \ldots, 1)
$$

such that the matrix function $N(\psi, \tau, \varepsilon) \in \mathfrak{N}$ is bounded in the complex domain $|\operatorname{Im} \psi|<\beta_{0} / 4, \tau \in D$ and the system (7.17) in the variables $\zeta, \psi, \tau$ takes the form

$$
\begin{aligned}
\dot{\zeta} & =\left[\tilde{\Lambda}(\tau, \varepsilon)+\varepsilon^{2} \tilde{B}(\psi, \tau, \varepsilon)\right] \zeta+\tilde{F}(\zeta, \psi, \tau, \varepsilon), \\
\dot{\psi} & =\tilde{\omega}(\tau, \varepsilon)+\tilde{F}^{\prime}(\zeta, \psi, \tau, \varepsilon), \\
\dot{\tau} & =\varepsilon,
\end{aligned}
$$

where $\tilde{B}(\psi, \tau, \varepsilon) \in \mathfrak{P}$, the functions $\tilde{B}, \tilde{F}, \tilde{F}^{\prime}$ are analytic and bounded for

$$
|\zeta| \leq \alpha_{0} / 4, \tau \in D,|\operatorname{Im} \psi|<\beta_{0} / 4,
$$

$\tilde{F}=O\left(|\zeta|^{2}\right), \quad \tilde{F}^{\prime}=O(|\zeta|)$.

The proof of this lemma uses the same approach as the proof of Lemma 7.1. One can write nonlinear differential equation for the required matrix $N$. Expansion into Fourier series leads to an infinite system of weakly nonlinear equations. Its solution is obtained via the iterations process of the Newton method. On each step of this process one should get a bounded solution of some linear system. This solution is obtained by using the method of contracting mappings.

Lemma 7.2 separates dynamics of variables $\zeta_{1}, \zeta_{2}$ from that of $\zeta_{3}, \ldots, \zeta_{n-1}$ in the linearized system. This allows to study dynamics of $\zeta_{1}, \zeta_{2}$ independently of details of dynamics of other variables and to proof Theorem 7 . 


\section{Final remarks}

1. The phenomenon of stability loss delay is related to exponentially small effects. Mathematical approaches used to study these effects may be found in [80, 64, 79] (continuous averaging), [17, 50] (Borel summation), and [31, 10] (holomorphic continuation of real dynamical objects).

2. Stability loss delay in PDEs and infinite-dimensional dynamical systems is considered in $[74,82,38,36,7,32,84]$.

3. Despite stability loss delay phenomenon can be destroyed by a very small noise it appears in applications. These applications include mechanics [57, 82], laser physics [24], chemistry [83], epidemiology [45], ecology[59], neural models [35, 22, 86, 87], modelling of a climate [23], biochemistry [19, 42, 43].

4. Control of stability loss delay is studied in [12, 70].

5. There are problems in which an equilibrium or a periodic trajectory of a fast system is neutrally stable (elliptic) in linear approximation for all values of slow variables. Then dynamical bifurcations with stability loss delay can be generated by nonlinear terms $[65,66]$.

6. Dynamical bifurcations in multifrequency systems may lead to stability loss delay phenomena [68, 61]. This important subject is at the beginning of its study.

\section{References}

[1] A.A. Andronov, Mathematical problems of the theory of auto-oscillations. I Allunion conference in oscillations. M., L.: GTTI, 1933, 32-72; Andronov A.A., Sob. soch. M.: 1956, 85-124.

[2] A.A. Andronov, A.A. Vitt, S.E. Khaikin, Theory of oscillators. Moscow: Fizmatgiz, 1937, translated from Russian in: Dover Publications, Inc., New York, 1987.

[3] V. I. Arnol'd, Proof of a theorem of A. N. Kolmogorov on the invariance of quasiperiodic motions under small perturbations of the Hamiltonian. Usp. Mat. Nauk, 18, No. 5, 13-40 (1963). Engl. transl.: Russ. Math. Surv. 18, No. 5, 9-36 (1963)

[4] V. I. Arnol'd, Supplementary chapters to the theory of ordinary differential equations. Nauka, Moscow (1978), 304 pp.; 2nd ed.: Geometric methods in the theory of ordinary differential equations. Research Centre "Regular and chaotic dynamics", Izhevsk (2000), 400 pp. Engl. transl.: Geometrical methods in the theory of ordinary differential equations. Grundlehren der Mathematischen Wissenschaften 250. Springer-Verlag, New York etc. (1988), 351 pp.

[5] V.I.Arnold, Catastrophe theory. Third edition. Springer-Verlag, Berlin (1992), IX, $79 \mathrm{pp}$.

[6] V.I. Arnold, V.V. Kozlov, A.I. Neishtadt, Mathematical aspects of classical and celestial mechanics. Springer-Verlag, Berlin, 2006. 
[7] D. Avitabile, M. Desroches, R. Veltz, M. Wechselberger, Local theory ot spatiotemporal canards and delayed bifurcations, SIAM J. Math. Anal. 52, 5703-5747 (2020)

[8] S. M. Baer, T. Erneux, J. Rinzel, The slow passage through a Hopf bifurcation: delay, memory effects and resonance, SIAM J. Appl. Math., 49, 1-17 (1989)

[9] C. Baesens, Slow sweep through a period-doubling cascade: Delayed bifurcations and renormalisation. Physica D, 53, 319-375 (1991)

[10] I. Baldomá, E. Fontich, M. Guardia, and T. Seara, Exponentially small splitting of separatrices beyond Melnikov analysis: Rigorous results. J. Differ. Equations 253, No. 12, 3304-3439 (2012).

[11] E. Benoit (ed.), Dynamic Bifurcations, Lecture Notes in Mathematics, Vol. 1493, Springer-Verlag, Berlin, 1991

[12] N. Berglund, Control of dynamic Hopf bifurcations, Nonlinearity, 13, 225-248 (2000)

[13] A. Bounemoura, B. Fayad, L. Niederman, Super-exponential stability for generic real-analytic elliptic equilibrium points. Adv. Math. 366 (2020), 107088, 30 pp.

[14] E. Benoit, J. F. Callot, F. Diener, M. Diener, Chasse au canard. Collectanea Mathematica, 31-32 (1-3), 37-119 (1981)

[15] A.D. Bryuno, Normalization of a Hamiltonian system near an invariant cycle or torus. (Russian) Uspekhi Mat. Nauk 44 (1989), no. 2(266), 49-78; translation in Russian Math. Surveys 44 (1989), no. 2, 53-89.

[16] J. L. Callot, Champs lents-rapides complexes à une dimension lente, Annales scientifiques de l'École Normale Supérieure, 4, 149-173 (1993)

[17] O. Costin, Asymptotics and Borel summability. Chapman and Hall/CRC Monographs and Surveys in Pure and Applied Mathematics, 141. CRC Press, Boca Raton, FL, 2009. xiv +250 pp.

[18] P. De Maesschalck, F. Dumortier, R. Roussarie, Canard Cycles: From Birth to Transition, Springer (2021), 429 pp.

[19] M. Desroches, B. Krauskopf, H. Osinga, The geometry of mixed-mode oscillations in the Olsen model for the peroxidase-oxidase reaction. Discrete Contin. Dyn. Syst. Ser. S, 2, no. 4, 807-827 (2009)

[20] F. Diener, M. Diener, Maximal delay. In: Dynamic Bifurcations (ed. E. Benoit), Lecture Notes in Mathematics, Vol. 1493. Springer-Verlag, Berlin, 71-86 (1991)

[21] R.B. Dingle, Asymptotic expansions: their derivation and interpretation. Academic Press, London-New York, 1973. xv+521 pp. 
[22] J. Drover, J. Rubin, J. Su, Jianzhong. B. Ermentrout, Analysis of a canard mechanism by which excitatory synaptic coupling can synchronize neurons at low firing frequencies. SIAM J. Appl. Math., 65, no. 1, 69-92 (2004)

[23] H. Engler, H. Kaper, T. Kaper, T. Vo, Dynamical systems analysis of the MaaschSaltzman model for glacial cycles. Physica D 359, 1-20 (2017)

[24] T. Erneux, P Mandel, Stationary, harmonic, and pulsed operations of an optically bistable laser with saturable absorber. II, Physical Review A, 18, 1902-1909 (1984)

[25] B. Fayad. Lyapunov unstable elliptic equilibria. arXiv:1809.09059

[26] B. Fayad, J.-P. Marco, and D. Sauzin. Attracted by an elliptic fixed point. Asterisque 416, 2020, 321-340, also: arXiv:1712.03001.

[27] N. Fenichel, Geometric singular perturbation theory for ordinary differential equations. J. Differential Equations 31, 1, 53-98 (1979)

[28] A. Fruchard, Existence of bifurcation delay: the discrete case. In: Dynamic Bifurcations (ed. E. Benoit), Lecture Notes in Mathematics, Vol. 1493. Springer-Verlag, Berlin, 87-106 (1991)

[29] A. Fruchard, Canards et râteaux, Annales de l'Institut Fourier, 42, 825-855 (1992)

[30] A. Fruchard, R. Schäfke, Bifurcation delay and difference equations, Nonlinearity, 16, $2199-2220(2003)$

[31] V.G. Gel'freich and V.F. Lazutkin Splitting of separatrices: Perturbation theory and exponential smallness. (English. Russian original) Russ. Math. Surv. 56, No. 3, 499-558 (2001); translation from Usp. Mat. Nauk 56, No. 3, 79-142 (2001).

[32] R. Goh, T. J. Kaper, T. Vo, Delayed Hopf bifurcation and space-time buffer curves in the Complex Ginzburg-Landau equation, arXiv:2012.10048 (2020)

[33] V.V. Golubev, Lectures on the analytic theory of differential equations. Moscow Leningrad, GITL, 436 pp. (1950) (Russian)

[34] Higher Transcendental Functions, v.2, McGraw-Hill, 1953

[35] E. Jakobsson, R. Guttman, Continuous Stimulation and Threshold of Axons: The Other Legacy of Kenneth Cole. In: The Biophysical Approach to Excitable Systems, Plenum, NY (1981)

[36] T. Kaper, T. Vo, Delayed loss of stability due to the slow passage through Hopf bifurcations in reaction-diffusion equations, Chaos, 28, no. 9, 091103, 9 pp. (2018)

[37] S. Karimov, Asymptotics of solutions of some classes of differential equations with small parameter multiplying derivatives, when there is change of stability of a stationary point in the fast motion plane, Differ. Equations, 21, 1136-1139 (1985) 
[38] E. Knobloch, R. Krechetnikov, Stability on time-dependent domains, J. Nonlinear Sci., 24, 493-523 (2014)

[39] A.M. Kovalev, A.N. Chudnenko, On the stability of the equilibrium of a twodimensional Hamiltonian system in case of equal frequencies, Dopov. Akad. Nauk Ukr. RSR, Ser. A, 1010-1013 (1977) (Ukrainian)

[40] V.V. Kozlov, S.D. Furta, Asymptotic expansions of solutions of strongly nonlinear systems of differential equations. Moscow Univ., Moscow, 244 pp. (1996)(Russian)

[41] V.V. Kozlov, D.V. Treschev, Billiards, a Genetic introduction to systems with impacts. Amer. Math. Soc. Transl. Ser., 171pp (1991)

[42] M. Krupa, A. Vidal, M. Desroches, F. Clément, Mixed-mode oscillations in a multiple time scale phantom bursting system, SIAM J. Appl. Dyn. Syst., 11, no. 4, $1458-1498$ (2012)

[43] C. Kuehn, P. Szmolyan, Multiscale geometry of the Olsen model and non-classical relaxation oscillations, J. Nonlinear Sci., 25, no. 3, 583-629 (2015)

[44] L.M. Lerman, A.P. Markova, On stability at the Hamiltonian Hopf bifurcation. Reg. and Chaotic Dyn., Vol.14, No 1, 148-162 (2009)

[45] M.Y. Li, W. Liu, C. Shan, Y. Yi, Turning points and relaxation oscillations cycles in simple epidemic models, SIAM J. Applied Math., 76, no. 2, 663-687 (2016)

[46] A.M. Lyapunov, The general problem of the stability of motion. Doctoral dissertation, University of Kharkov, Kharkov Mathematical Society, 250 pp. (1892) (Russian) English transl.: International Journal of Control 55, No. 3 (1992)

[47] A.P.Markeev and A.G.Sokolskii, Numeric study of stability of Lagrangian solutions in the elliptic restricted three-body problem. Appl. Math. and Mech. 1974, 38, No 1, 49-55. (in Russian)

[48] A.P. Markeev, Libration points in celestial mechanics and space dynamics. Moscow, Nauka, 1978.

[49] E.F. Mishchenko, N.Kh. Rozov, Differential Equations With Small Parameters and Relaxation Oscillations, New York-London: Plenum Press, 1980

[50] C. Mitschi, D. Sauzin, Divergent series, summability and resurgence. I. Monodromy and resurgence. Lecture Notes in Mathematics, 2153. Springer, [Cham], 2016. $\mathrm{xxi}+298 \mathrm{pp}$.

[51] A. I. Neishtadt, The separation of motions in systems with rapidly rotating phase. Prikl. Mat. Mekh. 48, No. 2, 197-204 (1984). Engl. transl.: J. Appl. Math. Mech. 48, No. 2, 133-139 (1985) 
[52] A.I. Neishtadt, Asymptotic study of stability loss of equilibrium under slow transition of two eigenvalues through imaginary axis. Uspekhi Mat. Nauk, 40, 5, 300-301 (1985) (Russian)

[53] A.I. Neishtadt, On stability loss delay for dynamical bifurcations, I. Differ.Uravn., 23, 12, 2060-2067 (1987) (Russian). English transl.: Differ. Equations, 23, 12, 1385$1390(1987)$

[54] A.I. Neishtadt, On stability loss delay for dynamical bifurcations, I|. Differ.Uravn., 24, 2, 226-233 (1988) (Russian). English transl.: Differ. Equations, 24, 2, 171-176 (1988)

[55] A.I. Neishtadt, On calculation of stability loss delay time for dynamical bifurcations. In: XI International Congress of Mathematical Physics (Paris, 1994) (ed. D. Yagolnitzer), International Press, Cambridge, MA, 280-287 (1995)

[56] A. I. Neishtadt, C. Simó, D. V. Treschev, On stability loss delay for a periodic trajectory. Progress in Nonlinear Differential Equations and Their Applications, 19, 253-278 (1996)

[57] A. I. Neishtadt, V. V. Sidorenko, Stability loss delay in a Ziegler system. J. Appl. Math. Mech., 61, 15-25 (1997)

[58] N.N. Nekhoroshev, An exponential estimate of the time of stability of nearly integrable Hamiltonian systems. II. Tr. Semin. Im. I. G. Petrovskogo 5, 5-50 (1979). Engl. transl.: Topics in modern mathematics, Petrovskii Semin. 5, 1-58 (1985)

[59] P.E. O'Keeffe, S. Wieczorek, Tipping phenomena and points of no return in ecosystems: beyond classical bifurcations. SIAM J. Appl. Dyn. Syst., 19, no. 4, 2371-2402 (2020)

[60] V.P. Palamodov, Stability of equilibrium in a potential field. Funkts. Anal. Prilozh. 11, No. 4, 42-55 (1977). Engl. transl.: Funct. Anal. Appl. 11, 277-289 (1978)

[61] I. Parasyuk, B. Repeta, Dynamical bifurcation of multifrequency oscillations in a fast-slow system. Electron. J. Differential Equations, Paper No. 233, 32 pp. (2016)

[62] H. Poincaré, Sur les proprietes des fonctions definies par les equations aux differences partielles. Paris.: G.V. 1879, Oeuvres de Henry Poincaré, Tome I, Paris: GauthierVillars. 1951, XLIX-CXXIX.

[63] L.S.Pontryagin, L.V. Rodygin, Periodic solution of a system of ordinary differential equations with a small parameter in the terms containing derivatives. Dokl. Akad. Nauk SSSR, 132, 537-540 (1960) (Russian); English transl.: Soviet Math. Dokl., 1 611-614 (1960)

[64] A.V. Pronin and D.V. Treschev Continuous averaging in multi-frequency slow-fast systems. (English) Regul. Chaotic Dyn. 5, No. 2, 157-170 (2000). 
[65] D. Rachinskii, K. Schneider, Delayed loss of stability in systems with degenerate linear parts, Zeitschrift $\mathrm{f}$ ur Analysis und ihre Anwendungen Journal for Analysis and its Applications, 22, no. 2, 433-453 (2003)

[66] D. Rachinskii, K. Schneider, Dynamic Hopf bifurcations generated by nonlinear terms, Differential Equations, 210, 65-86 (2005)

[67] J.-P. Ramis, R. Schäfke, Gevrey separation of fast and slow variables. Nonlinearity, 9, No. 2, 353-384 (1996)

[68] A. Samoilenko, I. Parasyuk, B. Repeta, Dynamical bifurcation of multifrequency oscillations in a fast-slow system. Ukraïn. Mat. Zh. 67, no. 7, 890-915 (2015); English translation: Ukrainian Math. J.67, no. 7, 1008-1037 (2015)

[69] M.A. Shishkova, Examination of one system of differential equations with a small parameter in highest derivatives. Dokl. Akad. Nauk SSSR, 209, 3, 576-579 (1973) (Russian). English transl.: Soviet Math. Dokl., 14, 2, 384-387 (1973)

[70] K.R. Schneider, E. Shchetinina, V.A. Sobolev, Control of integral manifolds loosing their attractivity in time, J. Math. Anal. Appl., 315, 740-757 (2006)

[71] Y. Sibuya, Sur reduction analytique d'un systeme d'equations differentiales ordinares lineaires content un parametre. J. Univ. Tokio, sect. 1, 10, 527-540 (1958)

[72] A.G. Sokolsky, On stability of an autonomous Hamiltonian system with two degrees of freedom in the case of equal frequencies. Prikl. Mat. Mech. 1974, Vol. 38, No. 5, 791-799 (in russian).

[73] A.G. Sokolsky, On stability of an autonomous Hamiltonian system with two degrees of freedom at a first order resonance resonance. Prikl. Mat. Mech. 1977, Vol. 41, No. 1, 24-33 (in russian).

[74] J. Su, On delayed oscillation in nonspatially uniform FitzHugh Nagumo equation, J. Differential Equations, 110, no. 1, 38-52 (1994)

[75] J. Su, Persistent unstable periodic motions, I, II. Journal of Mathematical Analysis and Applications, 198, 796-825, 199, 88-119 (1996)

[76] J. Su, Effects of periodic forcing on delayed bifurcations. J. Dynam. Differential Equations, 9, 56-625 (1997)

[77] J. Su, J. Rubin, D. Terman, Effects of noise on elliptic bursters, Nonlinearity, 17, 133-157 (2004)

[78] D. Treschev. Loss of stability in Hamiltonian systems that depend on parameters. (Russian) Prikl. Mat. Mekh. 56 (1992), no. 4, 587-596; translation in J. Appl. Math. Mech. 56 (1992), no. 4, 492-500

[79] D. Treschev, Splitting of separatrices for a pendulum with rapidly oscillating suspension point. Russ. J. Math. Phys. 5, No. 1, 63-98 (1997). 
[80] D. Treschev, O. Zubelevich, Introduction to perturbation theory of Hamiltonian systems. Springer-Verlag, Berlin, $\mathrm{x}+211$ pp. (2010)

[81] D. Treschev, Volume preserving diffeomorphisms as Poincaré maps for volume preserving flows, Russian Math. Surveys, 75:1 (2020), 187-189.

[82] L. Truskinovsky, A.Mielke, From discrete visco-elasticity to continuum rateindependent plasticity: rigorous results, Arch. Ration. Mech. Anal. 203, no. 2, 577-619 (2012)

[83] T. Tsotsis, R. Sane, T.Lindstrom, Bifurcation behavior of a catalytic reaction due to a slowly varying parameter, AIChE Journal, 34, no. 3, 383-388 (1988)

[84] J.C. Tzou, M.J. Ward, T. Kolokolnikov, Slowly varying control parameters, delayed bifurcations, and the stability of spikes in reaction-diffusion systems. Physica D, 290, 24-43 (2015)

[85] A.B.Vasil'eva, V.F. Butuzov, Asymptotic expansions of the solutions of singularly perturbed equations. Moscow, Nauka, 272 pp. (1973) (Russian)

[86] T. Vo, R. Bertram, M. Wechselberger, Multiple geometric viewpoints of mixed mode dynamics associated with pseudo-plateau bursting, SIAM J. Appl. Dyn. Syst., 12, no. $2,789-830$ (2013)

[87] T. Vo, Generic torus canards, Physica D, 356-357, 37-64 (2017) 